\title{
Aza-BODIPY Platform: Towards an Efficient Water-Soluble Bimodal Imaging Probe for MRI and Near-Infrared Fluorescence
}

Oceane Flores, Jacques Pliquett, Laura Abad Galan, Robin Lescure, Franck Denat, Olivier Maury, Agnès Pallier, Pierre-Simon Bellaye, Bertrand Collin, Sandra Même, Celia S. Bonnet, Ewen Bodio, Christine Goze Submitted date: 05/12/2019 - Posted date: 19/12/2019

Licence: CC BY-NC-ND 4.0

Citation information: Flores, Oceane; Pliquett, Jacques; Abad Galan, Laura; Lescure, Robin; Denat, Franck; Maury, Olivier; et al. (2019): Aza-BODIPY Platform: Towards an Efficient Water-Soluble Bimodal Imaging Probe for MRI and Near-Infrared Fluorescence. ChemRxiv. Preprint.

https://doi.org/10.26434/chemrxiv.11322860.v1

In this study, an original aza-BODIPY system comprising two $\mathrm{Gd}^{3+}$ complexes has been designed and synthesized for magnetic resonance imaging/optical imaging application, by functionalization of the boron center. This strategy enabled to obtain a positively-charged bimodal probe, which displays an increased water-solubility, optimized photophysical properties in the near-infrared region, and very promising relaxometric properties. The absorption and emission wavelengths are 705 and $741 \mathrm{~nm}$ respectively, with a quantum yield of around $10 \%$ in aqueous media. Moreover, the system does not produce singlet oxygen upon excitation, which would be toxic for tissues. The relaxivity obtained is high at intermediate fields (16.1 $\mathrm{mM}^{-1} . \mathrm{s}^{-1}$ at $20 \mathrm{MHz}$ and $310 \mathrm{~K}$ ) and competes with that of bigger or more rigid systems. A full relaxometric and ${ }^{17} \mathrm{O}$ NMR study and fitting of the data using the Lipari-Szabo approach showed that this high relaxivity can be explained by the size of the system and the presence of some small aggregates. These optimized photophysical and relaxometric properties highlight the potential use of such systems for future bimodal imaging studies.

File list (2)

inorg_chem_corr.docx (1.09 MiB) view on ChemRxiv • download file inorg_chem_corr.pdf (1.16 MiB) view on ChemRxiv - download file 


\section{Aza-BODIPY Platform: Towards an Efficient Water-Soluble Bimodal Imaging Probe for MRI and Near-Infrared Fluorescence}

Océane Florès ${ }^{\mathrm{a}}$, Jacques Pliquett ${ }^{\mathrm{a}}$, Laura Abad-Galan ${ }^{\mathrm{b}}$, Robin Lescure ${ }^{\mathrm{a}}$, Franck Denat ${ }^{\mathrm{a}}$, Olivier Maury ${ }^{\mathrm{b}}$, Agnès Pallier ${ }^{c}$, Pierre-Simon Bellaye ${ }^{d}$, Bertrand Collin ${ }^{a-d}$, Sandra Même ${ }^{c}$, Célia S. Bonnet ${ }^{c *}$, Ewen Bodio $^{a *}$, Christine Goze ${ }^{a *}$.

[a] CNRS, Université Bourgogne Franche-Comté, ICMUB UMR6302 - CNRS, F-21000 Dijon, France. [b] Univ Lyon, Ecole Normale Supérieure de Lyon, CNRS, Université Claude Bernard Lyon 1, Laboratoire de Chimie, UMR 5182, F-69342 Lyon, France.

[c] Centre de Biophysique Moléculaire, CNRS, Université d'Orléans, Rue Charles Sadron, 45071 Orléans Cedex 2, France.

[d] Centre Georges François Leclerc, Service de médecine nucléaire (plateforme d'imagerie et de radiothérapie précliniques), 1 rue Professeur Marion, BP77980, 21079 Dijon Cedex

\section{Abstract}

In this study, an original aza-BODIPY system comprising two $\mathrm{Gd}^{3+}$ complexes has been designed and synthesized for magnetic resonance imaging/optical imaging application, by functionalization of the boron center. This strategy enabled to obtain a positively-charged bimodal probe, which displays an increased water-solubility, optimized photophysical properties in the near-infrared region, and very promising relaxometric properties. The absorption and emission wavelengths are 705 and $741 \mathrm{~nm}$ respectively, with a quantum yield of around $10 \%$ in aqueous media. Moreover, the system does not produce singlet oxygen upon excitation, which would be toxic for tissues. The relaxivity obtained is high at intermediate fields $\left(16.1 \mathrm{mM}^{-1} \cdot \mathrm{s}^{-1}\right.$ at $20 \mathrm{MHz}$ and $\left.310 \mathrm{~K}\right)$ and competes with that of bigger or more rigid systems. A full relaxometric and ${ }^{17} \mathrm{O} N M R$ study and fitting of the data using the LipariSzabo approach showed that this high relaxivity can be explained by the size of the system and the presence of some small aggregates. These optimized photophysical and relaxometric properties highlight the potential use of such systems for future bimodal imaging studies.

\section{Introduction}

Imaging and especially molecular imaging has gained an increasing interest during these last years. Indeed, the insights in this field facilitate an earlier and more reliable diagnostic, enable the monitoring of the efficiency of a therapy, and even assist the surgeon during operations. ${ }^{1-6}$ Therefore, molecular imaging can benefit to a large number of pathologies. Moreover, this imaging approach also permits the discovery of novel biological targets or biomarkers and help to understand mechanisms of different diseases. Each molecular imaging technique has its own advantages and drawbacks and synergy is expected by association of two complementary modalities in a single molecular probe. ${ }^{7}$ This is particularly true when combining the two non-ionizing techniques: Magnetic Resonance Imaging (MRI) with Optical Imaging (OI). Indeed, MRI has an excellent spatial resolution and offers 3D anatomical and also functional information, which is ideal for in vivo imaging, but it suffers from low sensitivity. On the other hand, Ol exhibits high sensitivity and high microscopic resolution ( $<10 \mathrm{~nm}$ in the case of super resolved fluorescent microscopy), but suffers from poor tissue penetration. ${ }^{8}$ Another advantage of $\mathrm{Ol}$ is that it enables real time imaging. Moreover, more recently, despite its limited penetration depth, it has gained an increasing interest in the clinic for intraoperative fluorescence assisted surgery, where the use of fluorescence imaging particularly helps the surgeon to better delineate tumor margins, identify the lymph nodes invaded by cancer cells... ${ }^{9}$ 
In order to perform bimodal MRI/OI, there is an increasing interest in the conception of new efficient multimodal probes. Indeed, combination of these two imaging modalities into a single molecule assures the identical biodistribution compared to the combination of two separate imaging agents, and thus enables to perform a much more accurate diagnosis. The design of such bimodal probes is a real challenge, mainly because of the difference of sensitivity between MRI and OI. Therefore, the majority of the reported MRI/OI agents are based on functionalized polymers or nanoparticles. However small molecular bimodal agents were also reported and proved to be very effective. These agents combine fluorine atoms for ${ }^{19} \mathrm{~F}$ MRI or one, two, or more complexes, which are active in MRI, such as $\mathrm{Mn}^{2+}$ or $\mathrm{Gd}^{3+}$ complexes, and one fluorophore, which can be a dipyridophenazine, fluorescein, cyanine, rhodamine, quinoline, BODIPY... ${ }^{10-18}$

For in vivo optical imaging applications, the fluorophores need to absorb and emit in the spectral range, where biological tissues are more transparent and less scattering. This region, often called biological, transparency or theranostic window, corresponds to near infrared (NIR, as example NIR I corresponds to $650-900 \mathrm{~nm}) .{ }^{19-21}$ Development of NIR fluorophores is a hot topic because, despite the diversity of reported fluorophores, it seems that none of them is ideal for in vivo applications, and that most of them lack of chemical and photochemical stability, are poorly water soluble, low emissive, difficult to functionalize and their synthesis is time-consuming. ${ }^{22-25}$ Very recently, the azaBODIPY family attracted considerable attention because it may be one of the best candidates to tackle all these issues. These very stable BODIPY derivatives display a lower brightness than wellknown NIR cyanines such as ICG and IRDye ${ }^{\varpi} 800 \mathrm{CW}$ (Figure 1), but can be rapidly synthesized in large scale and are easily functionalized. ${ }^{26}$

Figure 1: structure of some optical imaging clinically used contrast agents and of Wazaby (watersoluble aza-BODIPY), we reported previously ${ }^{27}$

However, despite their potential, there are only very scarce reports of bimodal MRI/OI probes including (aza)-BODIPY. They have been used in combination with several fluorine atoms for ${ }^{19} \mathrm{~F} \mathrm{MRI},{ }^{17}$ or with two GdDTPA complexes, ${ }^{28}$ or GdDOTA complexes in a monomolecular system ${ }^{13}$ or in a polyrotaxane ${ }^{29}$. The sensitivity of ${ }^{19} \mathrm{~F}$ MRI remains lower than that of ${ }^{1} \mathrm{H}$ MRI. GdDTPA complexes are not the best candidates for in vivo use (vide infra) and the relaxivity of the BODIPY-GdDOTA system (vide infra) can certainly be optimized. This leaves room for improvement of such systems. It should 
also be noted that a bisGdDTPA-aza-BODIPY system was recently reported by Kim, Long and coll. to track in vivo phagocytic immune cells by MRI/OI bimodal imaging, highlighting the potential of such systems. $^{28}$

Despite their interesting properties, the limited use of BODIPY in bimodal imaging can certainly be explained by their poor water solubility, which is problematic for their use in vivo, and particularly for MRI. ${ }^{30-32}$ Several groups tethered water-solubilizing groups to the aza-BODIPY core, but even if sometimes it enabled in vitro and in vivo investigations, the resulting probes were still subject to significant aggregation phenomena (requiring the addition of surfactant). ${ }^{30,33,34}$ Very recently, some of us succeeded in designing fully water-soluble aza-BODIPY derivatives, called Wazaby (for watersoluble aza-BODIPY), thanks to the introduction of ammonium groups via boron functionalization (functionalizing boron induced aza-BODIPY faces hindrance, which prevented aggregation) (Figure 1). ${ }^{27}$ These probes were bioconjugated onto antibodies, were very stable in physiological media, and presented better in vivo optical properties than the Cy5, used as a control.

Building on this success, we decided to further investigate the potential of these Wazaby dyes and to conceive MRI/OI MOnomolecular Multimodal Imaging Probes (MOMIP). For this aim, we decided to attach two gadolinium complexes onto the boron-functionalized arms of the Wazaby scaffold, thanks to the strategy developed previously (Figure 2). Concerning the aza-BODIPY core, we chose an azaBODIPY bearing methoxy groups on the aromatic rings at the "south" of the molecule (also called proximal phenyl groups in aza-BODIPY chemistry) in order to shift the maximal absorption wavelength around $690-700 \mathrm{~nm}$. To maximize this effect (maximal absorption wavelength $>700 \mathrm{~nm}$ ) by creating a greater "push-pull" effect, bromine atoms were introduced on the aromatic rings at the "north" of the molecule (distal phenyl groups). It has to be noted that this functional group would enable further functionalization of the molecule in the future (extension of conjugation or introduction of a bioconjugatable handle if vectorization is needed). ${ }^{35}$

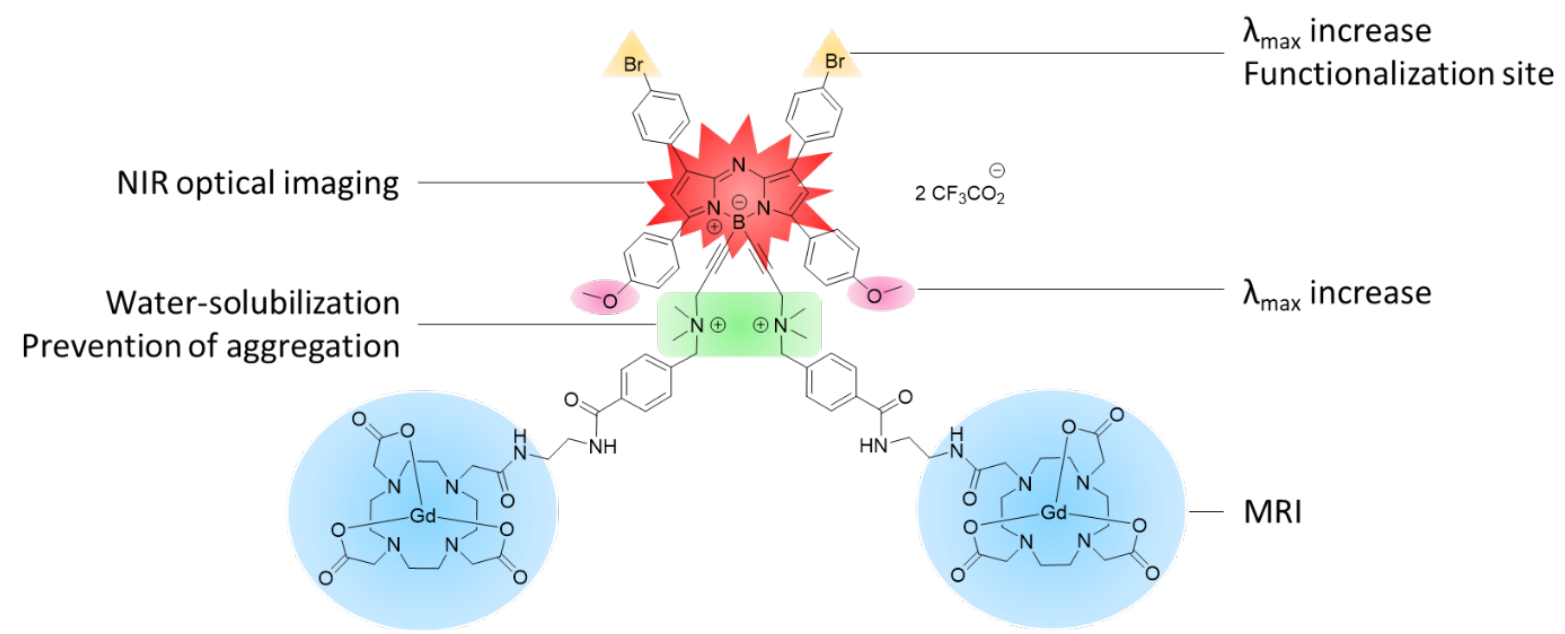

Figure 2 : Targeted bimodal probe and justification of the chosen functionalization

In MRI, DOTAREM ${ }^{\otimes}$ is one of the most clinically used contrast agents due to its high thermodynamic stability and kinetic inertness, which is essential for the safe use of the probe. ${ }^{36}$ Indeed, free gadolinium ions are highly toxic, and recently, complexes with lower kinetic inertness have been shown to increase the risk of nephrogenic systemic fibrosis (NSF), especially for patients having renal failure. ${ }^{37}$ As macrocyclic chelates are known to be more stable than linear ones, a DOTA derivative (DOTA monoamide or DOTAMA) was used for $\mathrm{Gd}^{3+}$ complexation. 


\section{Results and Discussion}

\section{Synthesis}

Aza-BODIPY 2 was synthesized at the gram scale according to the method we previously reported, ${ }^{27}$ starting by the synthesis of the aza-BODIPY $1,{ }^{38}$ followed by the substitution of the fluorine atoms on the boron center using the Grignard reagent of $\mathrm{N}, \mathrm{N}$-dimethylpropargylamine. The resulting intermediate platform was then alkylated by an excess of 4-(bromomethyl)benzoic acid in presence of $\mathrm{NaHCO}_{3}$ (Scheme 1) to give Wazaby5. Special attention was paid to the base employed as proton scavenger. A stronger base led to the formation of small amount of ester side product - alkylation of the carboxylate -, leading to a tricky purification.
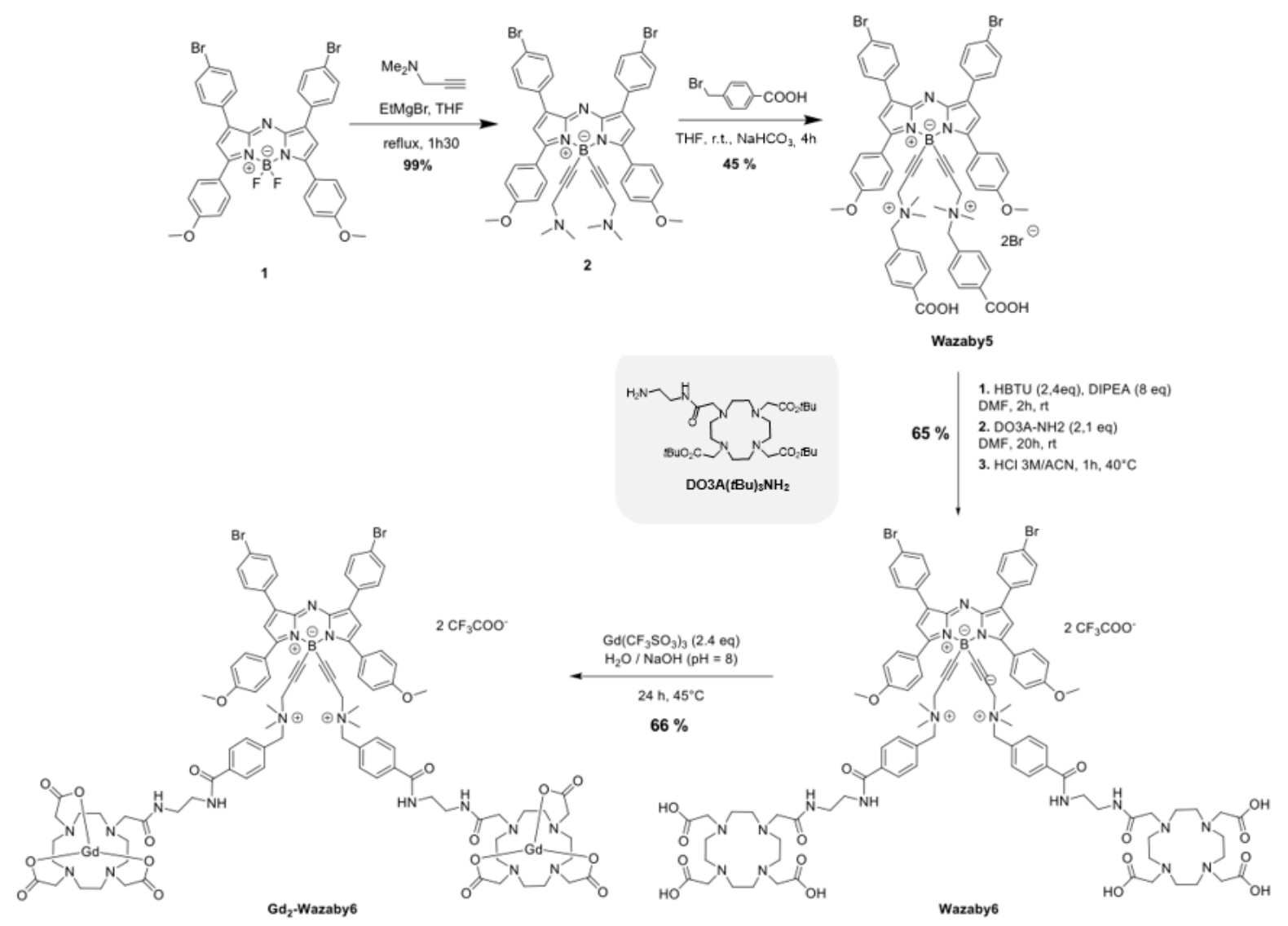

Scheme 1: synthetic route toward the bimodal probe $\mathrm{Gd}_{2}$-Wazaby6

Wazaby5 was reacted with a macrocyclic derivative $\left(\mathrm{DO} 3 \mathrm{~A}(\mathrm{tBu})_{3} \mathrm{NH}_{2}\right)$ in classic peptidic coupling reaction conditions and directly treated by $\mathrm{HCl} 3 \mathrm{M}$ in order to hydrolyze the protected tertiobutylester arms. The bis-DOTA derivative was then purified by semi-preparative HPLC to lead to the formation of pure Wazaby6. It is worth noting that the fluxionality of macrocycles implied to record NMR spectra at high temperature. A representation of variable temperature ${ }^{1} \mathrm{H}-\mathrm{NMR}$ of Wazaby6 is displayed in Figure S2.

Gadolinium was introduced into the two macrocycle cavities by reacting 2.4 eq of $\mathrm{Gd}\left(\mathrm{CF}_{3} \mathrm{SO}_{3}\right)_{3}$ salt with Wazaby6 in water in presence of sodium hydroxyde. The resulting homobimetallic complex $\mathbf{G d}_{2} \mathbf{2}^{-}$ Wazaby6 was characterized by high resolution mass spectrometry (HRMS), HPLC, elemental analysis and ionic chromatography but not NMR due to its paramagnetic character. 
As highlighted in the introduction, aza-BODIPY fluorophores display very interesting properties for in vivo optical imaging. However, in general, their main drawback is their poor solubility and their tendency to aggregate in water and in physiological media, despite the introduction of solubilizing groups. Most of the time, imaging experiments are performed in the presence of surfactants like Cremophor EL or castor oil. ${ }^{39-42}$ Therefore, it was crucial for us to investigate the photophysical properties of our bimodal compounds in organic solvent (DMF, DMSO), as well as in PBS (PhosphateBuffered Saline), without adding any surfactant.

Table 1: photophysical data of Wazaby5, Wazaby6, and $\mathbf{G d}_{2}$-Wazaby6 in different solvents at $298 \mathrm{~K}$ at $2 \mu \mathrm{M}\left(\lambda_{\text {exc }}=670 \mathrm{~nm}\right)$.

\begin{tabular}{|c|c|c|c|c|c|}
\hline Compound & Solvent & $\begin{array}{c}\lambda_{\max , \text { Abs }} \\
{[\mathrm{nm}]}\end{array}$ & $\begin{array}{c}\varepsilon_{\lambda \max } \\
{\left[\mathrm{M}^{-1} \mathrm{~cm}^{-1}\right]}\end{array}$ & $\begin{array}{c}\lambda_{\max , \mathrm{Em}} \\
{[\mathrm{nm}]}\end{array}$ & $\varphi_{\mathrm{F}}[\%]^{*}$ \\
\hline \multirow{2}{*}{ Wazaby5 } & DMSO & 704 & 74,000 & 739 & 29 \\
\hline \multirow{3}{*}{ Gd $_{2}$-Wazaby6 } & DMSO & 705 & 82,000 & 741 & 15 \\
\cline { 2 - 6 } & PBS & 705 & 46,000 & 741 & 10 \\
\cline { 2 - 6 } & DMSO & 705 & 78,000 & 739 & 22 \\
\cline { 2 - 6 } & PBS & 705 & 41,000 & 741 & $\mathbf{9}$ \\
\cline { 2 - 6 } & water & 705 & 49,000 & 741 & 10 \\
\hline
\end{tabular}

* reference: aza-BODIPY $3\left(\varphi_{\mathrm{F}}=36 \% \text { in chloroform, } 298 \mathrm{~K}, \lambda_{\mathrm{exc}}=670 \mathrm{~nm}\right)^{43}$

The photophysical data of Wazaby5, Wazaby6, and the related bimetallic complex $\mathbf{G d}_{2}$-Wazaby6 were studied in DMSO, DMF, and PBS, and results are depicted in Table 1, Figure 3, and Table S2. The three compounds display comparable absorption and emission profiles, with absorption wavelength at $\approx 705 \mathrm{~nm}$ and emission maxima at $\approx 740 \mathrm{~nm}$. Interestingly, the introduction of the two macrocycles as well as the complexation of the two gadolinium atoms had almost no impact on the photophysical properties of the fluorophore.

When comparing Wazaby6 and $\mathrm{Gd}_{2}$-Wazaby6 in DMSO and PBS, we can notice that the absorption, emission and excitation profiles of the compounds are comparable in both media (Figure 3), even if we can observe that absorption band is slightly larger than excitation one between 600 and $650 \mathrm{~nm}$ in PBS. It suggests that very limited aggregation occurs in PBS. If these spectra are compared to the ones of Kim's and Long's system, ${ }^{28}$ it clearly highlights the interest of boron-functionalization for limiting aggregation phenomenon, which is really frequent for aza-BODIPY fluorophores. Interestingly, the quantum yield efficiency remains comparable both in organic solvents (around 20\%) and in PBS (around 10\%). Such values are good for NIR emitters and are clearly enough to undertake future OI experiments.

It is well known that the presence of "heavy atoms" induces an increase of the spin-orbit coupling and therefore promotes the generation of singlet oxygen. ${ }^{32,44}$ However, this effect is generally more substantial when the heavy atoms occupy a position in the core region of the aza-BODIPY. In the present study, a non-toxic probe is under scope, thus, the production of singlet oxygen needs to be controlled. In order to test if toxic singlet oxygen is generated, the probes were dissolved in a nonprotic solvent (DMF) for feasible observation of the phosphorescence band of singlet oxygen at $1270 \mathrm{~nm}$, as protic solvents will efficiently quench its emission. ${ }^{45}$ In every case this band was found to be absent, which confirms that neither Wazaby6 nor $\mathbf{G d}_{2}$-Wazaby6 produce singlet oxygen, when 
irradiated (see supporting information for details). Moreover, preliminary antiproliferative tests on HepG2 cells (Human hepatocellular carcinoma, ATCC HB-8065" ${ }^{\text {mW }}$ ) confirmed that Gd $_{2}$-Wazaby6 do not display significant toxicity $\left(\mathrm{IC}_{50}\right.$ at $\left.24 \mathrm{~h}>100 \mu \mathrm{M}\right)$. Additional experiments will be performed in the future to ascertain this aspect.
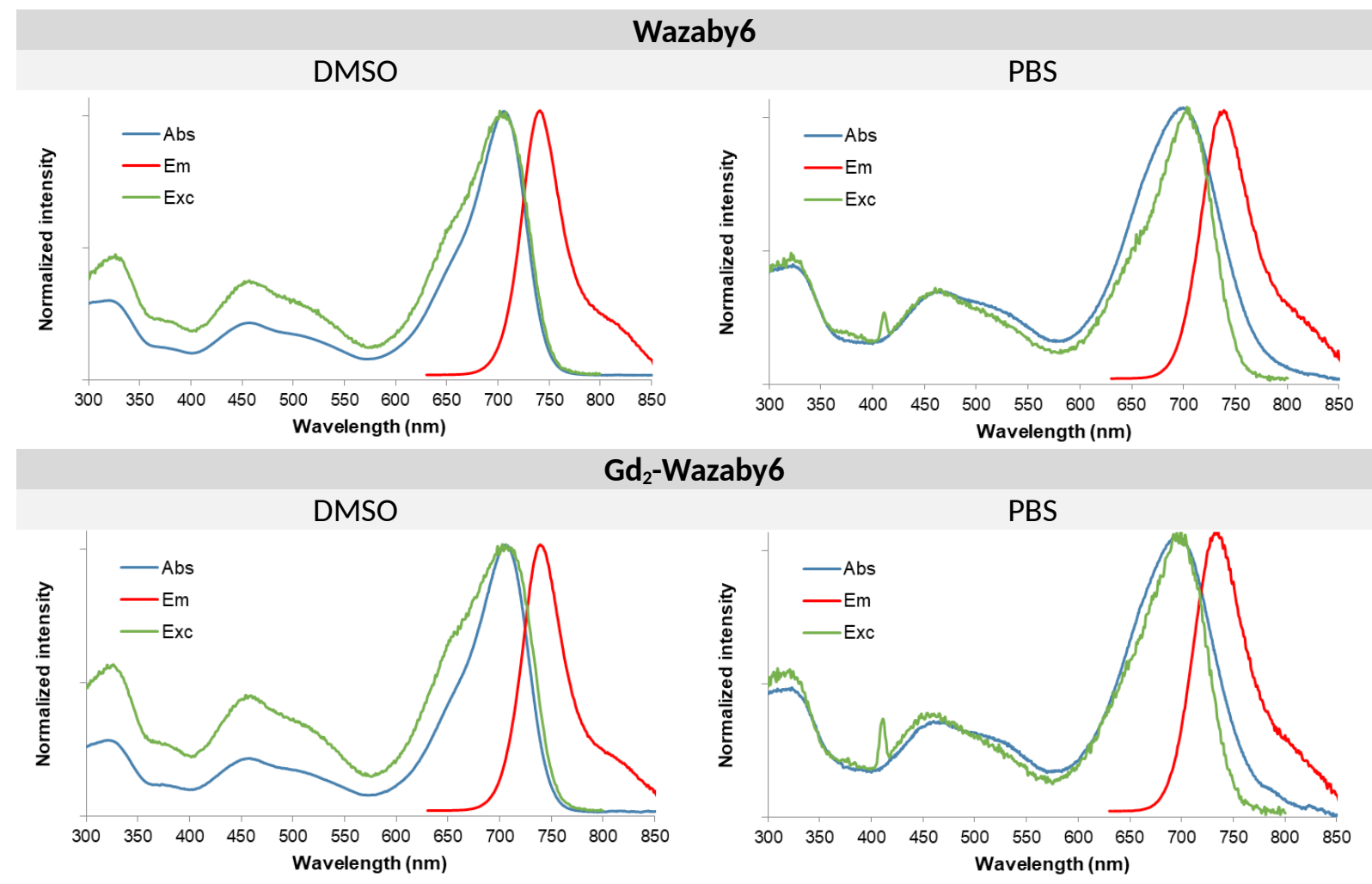

Figure 3: Normalized absorption, emission, and excitation spectra of Wazaby6 and $\mathrm{Gd}_{2}$-Wazaby6 at $298 \mathrm{~K}\left(\lambda_{\text {ex }}=620 \mathrm{~nm}\right.$ for emission spectra, detection at $\lambda_{e m}=820 \mathrm{~nm}$ for excitation spectra)

Relaxivity studies

Given the good solubility of the system, relaxivity measurements could be undertaken to characterize the efficiency of the system as an MRI probe. To this aim, NMRD (Nuclear Magnetic Resonance Dispersion) profiles were measured in water at $0.64 \mathrm{mM}$ at $\mathrm{pH}=7.2$ in the field range $40 \mathrm{kHz}-400$ $\mathrm{MHz}$ at two different temperatures $(298 \mathrm{~K}$ and $310 \mathrm{~K}$ ) as illustrated in Figure 4.

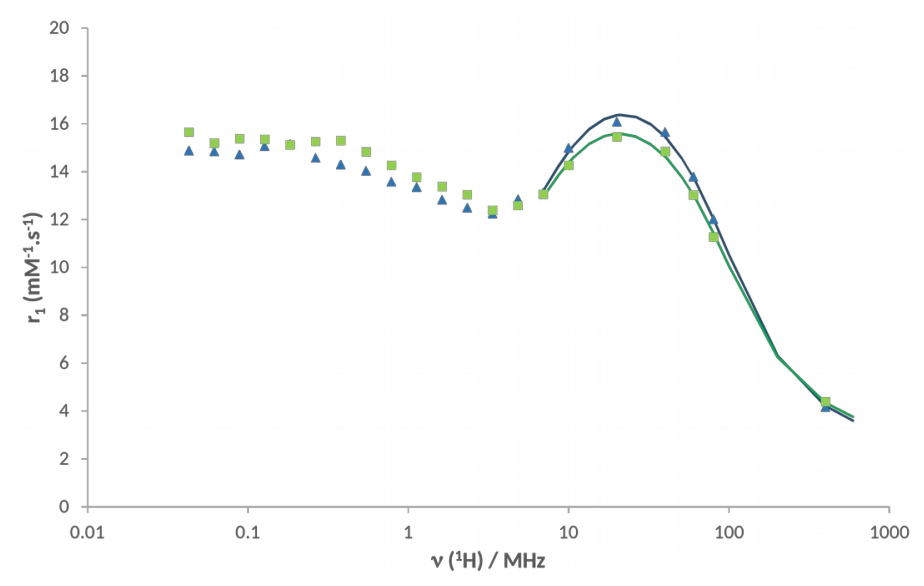


Figure 4: ${ }^{1} \mathrm{H}$ NMRD profile of $\mathrm{Gd}_{2}$-Wazaby6 $0.64 \mathrm{mM}$ in water, $\mathrm{pH}=7.2$ at $298 \mathrm{~K}(\square)$ and $310 \mathrm{~K}(\square)$. The lines represent the best fit to the SBM theory.

Interestingly, the temperature dependence of the system shows that we are in an intermediate regime where the rotation of the system starts to limit relaxivity rather than the water exchange rate. Indeed, the $37^{\circ} \mathrm{C}$ relaxivities are very close and even higher than those at $25^{\circ} \mathrm{C}$. Typically, when the rotation is the limiting parameter (as observed for small molecular systems), relaxivity is decreasing when increasing temperature, whereas when the water exchange rate is limiting, relaxivity is increasing with increasing temperature. This result is also in accordance with the characteristic hump of slowly-rotating species visible on the NMRD profiles at intermediate fields $(20-80 \mathrm{MHz})$. As this system remains quite flexible, we decided to investigate the relaxivity as a function of the $\mathrm{Gd}^{3+}$ concentration. Relaxivities were measured in the $\mathrm{Gd}^{3+}$ concentration range $0.1-4 \mathrm{mM}(0.05-2 \mathrm{mM}$ for the system) both in water at $\mathrm{pH}=7.2$ and in PBS at $\mathrm{pH}=7.4$, and the results are presented in Figure 5. At the concentration used for measuring relaxivities (0.64 $\mathrm{mM}$ of $\mathbf{G d}_{2}$-Wazaby6), we can see that some aggregation occurs both in water and PBS probably due to interactions between the azaBODIPY units. This is in accordance with the photophysical studies, highlighting small aggregation, but which doesn't prevent water solubility. The aggregation is favored in PBS, which can be explained by additional electrostatic interactions between the $\mathbf{G d}_{2}$-Wazaby6 cation (positive charge) and PBS (minus charge). This phenomenon certainly explains the temperature dependency of this otherwise quite flexible system.

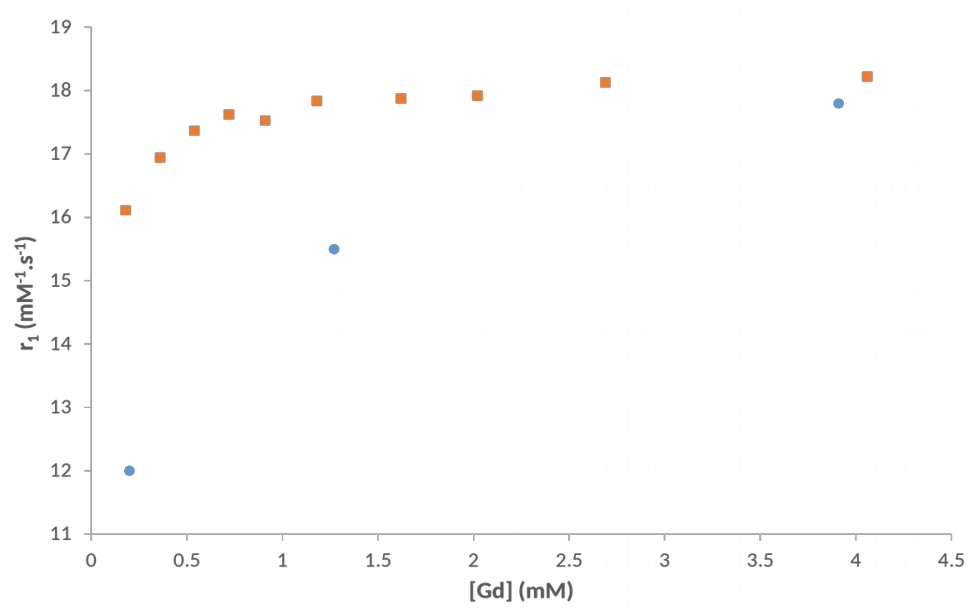

Figure 5: Relaxivity of the complex as a function of $\mathrm{Gd}^{3+}$ concentration at $298 \mathrm{~K}, 20 \mathrm{MHz}$, in water at $p H=7.2(\bullet)$ and in PBS at $p H=7.4(\boldsymbol{\square})$.

The relaxivity of the system at $0.64 \mathrm{mM}$ in water and at $20 \mathrm{MHz}, 310 \mathrm{~K}$ is $16.1 \mathrm{mM}^{-1} \cdot \mathrm{s}^{-1}$. As a comparison, and as expected due to the aggregation phenomenon, it is considerably higher than that of the mononuclear GdDOTA-BODIPY complex $\left(3.9 \mathrm{mM}^{-1} \cdot \mathrm{s}^{-1}\right)^{13}$ and the relaxivity of the Bis-GdDTPAaza-BODIPY system is $13.3 \mathrm{mM}^{-1} \cdot \mathrm{s}^{-1}$ at $60 \mathrm{MHz}$ and room temperature ${ }^{28}$ (see Figure $\mathrm{S} 10$ for structures). The relaxivity is in the same order of magnitude than a multimodal polyrotaxane comprising cyclodextrins bearing GdDOTAMA or BODIPYs $\left(r_{1}=18.60 \mathrm{mM}^{-1} \cdot \mathrm{s}^{-1}\right)^{29}$ or a porphyrinic system DPP-ZnPGdDOTA (see Figure S10; $r_{1}=17.9 \mathrm{mM}^{-1} . \mathrm{s}^{-1}$ ) presenting also some aggregation. ${ }^{46}$ Finally, the relaxivity compares well with those of nanosized objects such as $\mathrm{MOFs}^{47}$ and micelles ${ }^{48,49}$.

In order to gain more insight into the parameters governing relaxivity, ${ }^{17} \mathrm{O}$ measurements as a function of temperature have been performed. Variable temperature ${ }^{17} \mathrm{O} T_{2}$ measurements give access to the water exchange rate, $k_{\mathrm{ex}}$. The ${ }^{17} \mathrm{O} T_{1}$ data are determined by dipole-dipole and quadrupolar relaxation mechanisms and provide information about the rotational correlation time, $\tau_{R}$. The ${ }^{17} \mathrm{O}$ chemical shifts give indication of the number of water molecules directly coordinated to $\mathrm{Gd}^{3+}$, q. Longitudinal, transverse ${ }^{17} \mathrm{O}$ relaxation rates and chemical shifts were measured as a function of the temperature on aqueous solution of $\mathbf{G d}_{2}$-Wazaby6 at $2.39 \mathrm{mM}$, and on a diamagnetic reference 
$\left(\mathrm{HClO}_{4}, \mathrm{pH}\right.$ 3.3) at 9.4 T. Due to the concentration used for the study, ${ }^{17} \mathrm{O}$ chemical shifts and $\mathrm{T}_{1}$ values were too close to those of the reference to yield reliable data. Only ${ }^{17} \mathrm{O} \mathrm{T}_{2}$ data were analyzed and are presented in Figure 6.

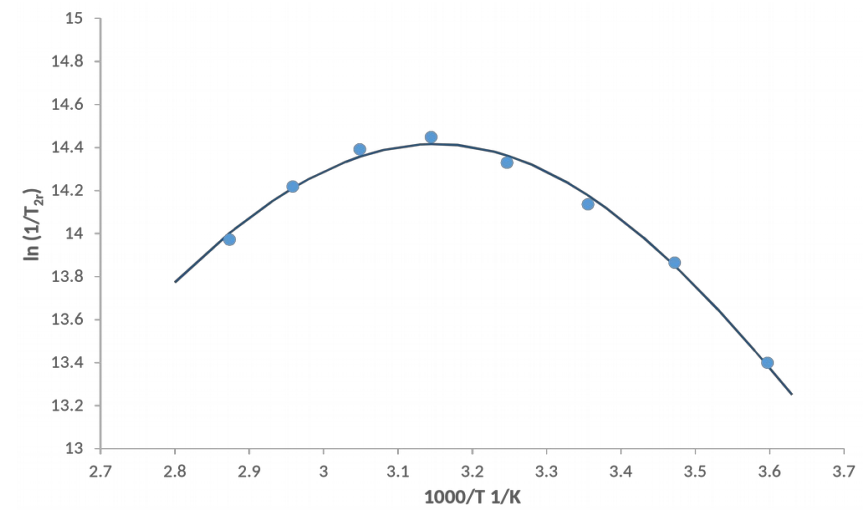

Figure 6: Temperature dependence of the reduced ${ }^{17} \mathrm{O}$ transverse relaxation rates of $\mathrm{Gd}_{2}$-Wazaby6

$(2.39 \mathrm{mM})$ at $9.4 \mathrm{~T}$. The continuous curve represents the best fit to the experimental data points.

The reduced ${ }^{17} \mathrm{O}$ transverse relaxation rates first increase (up to ca. $318 \mathrm{~K}$ ), then decrease with increasing temperature indicating that the complex is in the slow kinetic region at low temperatures and in the fast exchange region at higher temperatures. In the slow kinetic region, $1 / T_{2 r}$ is directly determined by the exchange rate constant $k_{\mathrm{ex}}$, whereas in the fast exchange region, it is determined by the transverse relaxation rate of the coordinated water oxygen, $1 / \mathrm{T}_{2 \mathrm{~m}}$, which is in turn influenced by the water exchange rate, $k_{\mathrm{ex}}$, the longitudinal electronic relaxation rate, $1 / \mathrm{T}_{1 \mathrm{e}}$, and the scalar coupling constant, $A / \hbar$.

Due to the different concentrations used for the ${ }^{17} \mathrm{O}$ NMR $\left(4.78 \mathrm{mM}\right.$ of $\mathrm{Gd}^{3+}$ ) and NMRD samples $\left(1.27 \mathrm{mM}\right.$ of $\left.\mathrm{Gd}^{3+}\right)$ and the aggregation process previously evidenced, the ${ }^{17} \mathrm{O}$ NMR and NMRD data have been analysed separately according to the Solomon-Bloembergen-Morgan theory.

Table 2: Best-fit parameters obtained from the independent fitting of the ${ }^{1} \mathrm{H}$ NMRD profiles to the SBM theory, including the Lipari-Szabo approach to describe internal flexibility and the transverse ${ }^{17} \mathrm{O} N M R$ relaxation rates as a function of temperature (Figure 6) at $9.4 \mathrm{~T}$. The number in brackets represents the error on the last digit.

\begin{tabular}{|c|c|c|c|c|}
\hline Parameters & $\begin{array}{c}\text { Gd }_{2-} \\
\text { Wazaby6 }\end{array}$ & [DPP-ZnP-GdDOTA] $^{-c}$ & $\begin{array}{c}\text { GdDOTAC }_{12} \\
\text { (micellar form) }^{\mathrm{d}}\end{array}$ & Zn-LZF2Gd \\
\hline$r_{1}\left(m^{-1} \mathrm{~s}^{-1} ; 20 \mathrm{MHz}, 37^{\circ} \mathrm{C}\right)$ & 16.1 & 17.9 & $17.9^{\mathrm{b}}$ & 15.8 \\
\hline$q^{a}$ & 1 & 1 & 1 & 1 \\
\hline$k_{\mathrm{ex}}^{298}\left(10^{6} \mathrm{~s}^{-1}\right)$ & $2.81[ \pm 0.08]$ & 4.1 & 4.9 & 1.6 \\
\hline$\Delta H^{ \pm}\left(\mathrm{kJ} . \mathrm{mol}^{-1}\right)$ & $40[ \pm 1]$ & 49.8 & 52 & 40 \\
\hline$\Delta S^{\neq}\left(\mathrm{J} . \mathrm{K}^{-1} \cdot \mathrm{mol}^{-1}\right)$ & $13[ \pm 3]$ & - & +67 & - \\
\hline$E_{1}\left(\mathrm{~kJ} . \mathrm{mol}^{-1}\right)$ & $7[ \pm 4]$ & 40 & 20 & 20 \\
\hline$\tau_{1}^{298}(\mathrm{ps})$ & $111[ \pm 9]$ & 245 & 430 & 145 \\
\hline$E_{\mathrm{g}}\left(\mathrm{kJ} \cdot \mathrm{mol}^{-1}\right)$ & $5 \pm[1.5]$ & 14 & 35 & 13 \\
\hline$\tau_{\mathrm{g}}^{298}(\mathrm{ps})$ & $1840[ \pm 70]$ & 2640 & 1600 & 1160 \\
\hline$S^{2}$ & $0.30[ \pm 0.01]$ & 0.26 & 0.23 & 0.64 \\
\hline
\end{tabular}

${ }^{a}$ fixed in the fit; ${ }^{\mathrm{b}}$ at $60 \mathrm{MHz}$ and $298 \mathrm{~K} ;{ }^{\mathrm{c}}$ From ref ${ }^{46} ;{ }^{\mathrm{d}}$ From ref ${ }^{48} ;{ }^{\mathrm{e}}$ From ref ${ }^{50}$

In the fitting procedure, the number of water molecules directly coordinated to $\mathrm{Gd}^{3+}$ was fixed to 1 as it is expected for GdDOTAMA complexes. In the fit of the proton relaxivities, the water exchange parameters were fixed to values obtained in the ${ }^{17} \mathrm{O}$ NMR study. The rotational dynamics could be only described by applying the Lipari-Szabo approach. In this approach, that separates local and global motions, characterized by the local and global rotational correlation times, $\tau_{1}^{298}$ and $\tau_{\mathrm{g}}{ }^{298}$, 
respectively, and a model independent order parameter, $S^{2}$. It reflects the degree of spatial restriction of the local motion with respect to the global motion. Its value ranges from 0 to 1 , with $S^{2}=0$ if the internal motions are isotropic, and $\mathrm{S}^{2}=1$ if the internal motions are completely restricted.

The fitting has been restricted to frequencies above $6 \mathrm{MHz}$ for NMRD data as at low magnetic fields the SBM theory fails in describing electronic parameters and rotational dynamics of slowly rotating species. Details of the analysis and the equations used in the fit are given in the Supporting Information, and the most important parameters obtained are listed in Table 2. A complete list of the parameters fitted for our system is also given Table S1.

For comparison, three other systems have been presented within the table (Table 2): a porphyrinic system with a GdDOTAGA appended (DPP-ZnP-GdDOTA); a GdDOTAC ${ }_{12}$ complex in its micellar form, and a 30 aminoacid peptides derived from a $\mathrm{Zn}$-finger peptide and bearing a GdDOTAMA complex in a folded form (Zn-bound form; Zn-LZF2Gd). They have been chosen because: (1) they have been all analyzed using the Lipari Szabo approach; (2) the porphyrinic system has a similar molecular weight as our system and shows some small aggregation as well; (3) the GdDOTAC $_{12}$ is in its micellar form which could "mimick" an aggregated state; (4) the peptidic system has a similar coordination sphere for $\mathrm{Gd}^{3+}$ than our system and the relaxivity behavior as a function of temperature is similar (relaxivity limited by rotation rather than water exchange rate).

The fitting of the ${ }^{17} \mathrm{O}$ data yields a value of $k_{\mathrm{ex}}$ of $2.81 .10^{6} \mathrm{~s}^{-1}$, which is lower than that of GdDOTA ( $k_{\mathrm{ex}}=$ 4.1.10 $\mathrm{s}^{-1}$ ) as expected when a carboxylate function is replaced by an amide function, and in the same range than other GdDOTAMA complexes $\left(\mathrm{ca} .2 .10^{6} \mathrm{~s}^{-1}\right){ }^{51}$ It suggests that the aggregation process, which certainly occurs through the BODIPY moiety, does not affect the water exchange rate of the system. The activation entropy calculated is also positive and in the same order of magnitude as that of complexes possessing similar coordination spheres. The water exchange mechanism has not been directly assessed (it is beyond the scope of this study), but the activation entropy calculated gives an indication of a dissociative mechanism as expected for nine-coordinated $\mathrm{Gd}^{3+}$ complexes. In dissociative mechanism, the steric crowding is of primary importance as the determining step is the dissociation of the water molecule. A highly-crowded environment will favour this dissociative activation step. As carboxylate functions are more coordinating than amide function, they imply more constraints explaining why GdDOTA complexes have higher water exchange rates than GdDOTAMA complexes.

The local and global correlation times of the system are within the same range as those of similar systems giving rise to similar relaxivities as shown in Table 2 . The low value of $S^{2}$, comparable to those of DPP-ZnP-GdDOTA or the micellar system, shows that the motion of the $\mathrm{Gd}^{3+}$-coordinated water proton vector is largely decoupled from the overall motion of the aggregates. This can be explained by the quite flexible chain between the Aza-BODIPY core and the $\mathrm{Gd}^{3+}$ complexes. If we look in more detail, the global correlation time of the system correlates well with that of the micellar system, or that of DPP-ZnP-GdDOTA having a similar molecular weight and presenting some aggregation. It is slightly higher than that of the peptidic system with a higher molecular weight but which is not in an aggregated form. All this confirms that some aggregation occurs for our system. The value of the local correlation time, consistent with that of a small $\mathrm{Gd}^{3+}$ complex, corroborates the flexibility around the $\mathrm{Gd}^{3+}$ complexes and shows that the aggregation certainly occurs through the aza-BODIPY core.

Finally, the temperature dependence of the relaxivity of our system is related to the low water exchange rate of the system together with a slow motion explained by the size of the complex and the presence of some aggregation. The same behavior is not observed with the porphyrinic system due to the difference in the water exchange rates (explained by the different $\mathrm{Gd}^{3+}$ coordination spheres). On the contrary, the peptidic system presents the same (even exacerbated) temperature behavior, which is explained by a more rigid system with slightly lower water exchange rate, despite 
the same $\mathrm{Gd}^{3+}$ coordination sphere. To sum up, the high relaxivity observed is due to the size of the system and the presence of some small aggregates.

Preliminary imaging studies on phantom and measurements in HSA

Phantom images of the system $\mathbf{G d}_{2}$-Wazaby6 have been recorded both in MRI and in fluorescence to highlight its potential.

$\mathrm{T}_{1}$-weighted images were recorded at $7 \mathrm{~T}$, and show a greater contrast compared to the commercial GdDOTA at the same concentration $(150 \mu \mathrm{M})$ (Figure 7).

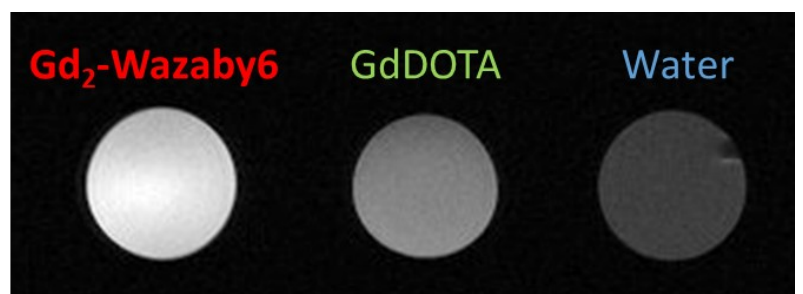

Figure 7: $T_{1}$-weighted MRI phantom images of $\mathrm{Gd}_{2}$-Wazaby6 $(150 \mu \mathrm{M})$, GdDOTA (150 $\left.\mu \mathrm{M}\right)$ and water (reference) at $7 \mathrm{~T}$ using spin-echo sequence with $T E=50 \mathrm{~ms}$ and $T R=500 \mathrm{~ms}$.

Fluorescence images were recorded thanks to an optical imager for in vivo experiments (IVIS Lumina III, Perkin Elmer, Figure 8). Gd $_{2}$-Wazaby6 was imaged at $150 \mu \mathrm{M}$ (the concentration used for MRI imaging), $15 \mu \mathrm{M}$, and $1.5 \mu \mathrm{M}$ in water; Aza-BODIPY 3 was imaged at $150 \mu \mathrm{M}$ as a control. The first observation that can be made is the strong fluorescence of $\mathbf{G d}_{2}$-Wazaby6 with respect to Aza-BODIPY 3 (3 is not fluorescent due to its insolubility in water). Additionally, it is worth noting that $\mathrm{Gd}_{2}$-Wazaby6 displays higher fluorescence at $15 \mu \mathrm{M}$ than at $150 \mu \mathrm{M}$, which corroborates the fact that some aggregation phenomenon can occur at high concentration. Moreover, we were pleased to note that a significant signal can be observed up to $850 \mathrm{~nm}$, especially when exciting the molecule at 700 or $720 \mathrm{~nm}$, meaning that $\mathrm{Gd}_{2}$-Wazaby6 can be detected wherever we want in biological window.

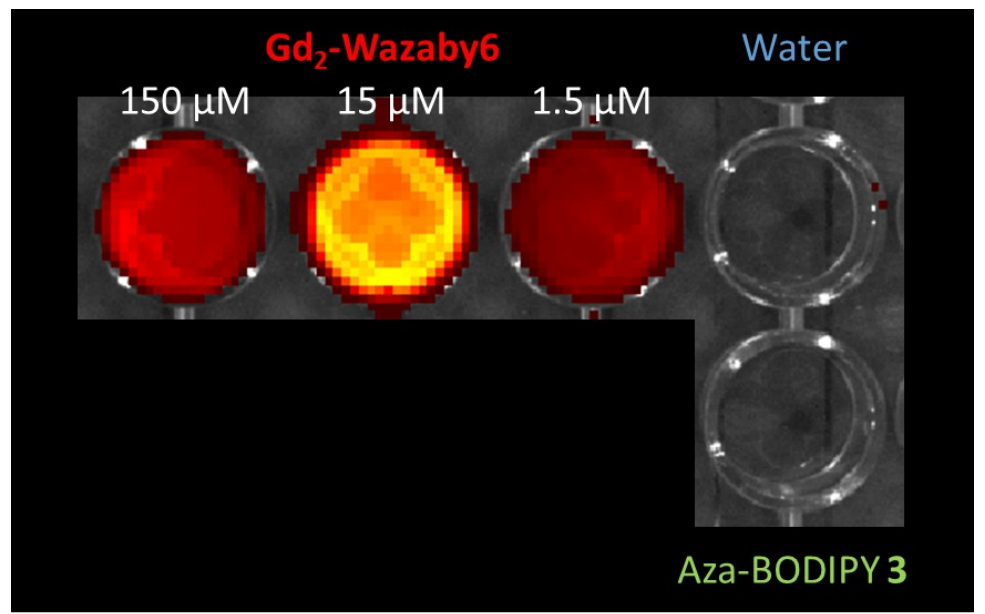

Figure 8: Phantom optical images of of $\mathrm{Gd}_{2}$-Wazaby6 (150 $\mu \mathrm{M}, 15 \mu \mathrm{M}$, and $\left.1.5 \mu \mathrm{M}\right)$, Aza-BODIPY 3 $(150 \mu \mathrm{M})$, and water (reference) excitation at $660 \mathrm{~nm}$ and detection at $710 \mathrm{~nm})$.

The relaxivity and the optical emission properties of the system have also been measured in the presence of $0.6 \mathrm{mM}$ of Human Serum Albumin (HSA) (physiological concentration) in order to simulate biological environment. Concerning the relaxivity of $\mathbf{G d}_{2}$-Wazaby6 (at $145 \mu \mathrm{M}$ ), a $14 \%$ increase is observed in water at $\mathrm{pH}=7.3,60 \mathrm{MHz}$, and $25^{\circ} \mathrm{C}$, in the presence of HSA. This increase is explained by the formation of a supramolecular adduct between the probe and HSA as commonly 
observed for hydrophobic systems. Concerning the optical properties, the emission of Gd2-Wazaby6 (at $2 \mu \mathrm{M}$ ) increased by $230 \%$ in presence of HSA, probably due to the more lipophilic environment. The improved properties of Gd2-Wazaby6 for both MRI and OI in presence of HSA are reassuring regarding its efficiency in real biological media.

These different preliminary studies are very promising for the future use of our probe for in vivo bimodal imaging.

\section{Conclusion}

For the first time, a monomolecular multimodal imaging probe for MRI and Near-Infrared Fluorescence $\left(\mathbf{G d}_{2}\right.$-Wazaby6) was synthesized thanks to a boron functionalization of an aza-BODIPY. It was fully characterized by usual spectroscopic techniques. The boron functionalization allows good solubility of the system both in water and PBS and enables full photophysical and relaxometric characterizations. Interestingly, the quantum yield of fluorescence of this bimodal system is around $10 \%$ in PBS, which is particularly high for a near infrared fluorophore. Moreover, this probe does not induce the production of singlet oxygen, which could be toxic for biological tissue. The relaxivity observed for $\mathbf{G d}_{2}$-Wazaby6 is high and can be explained both by the size of the system and the presence of some small aggregation process, as also observed in the optical imaging studies. Despite these aggregates the system remains very soluble in water and in biological media, and could be studied without the presence of surfactant as often needed with BODIPY derivatives. Those small aggregates do not imply important fluorescence quenching and enable to achieve high efficiency in MRI (in future work, it will be worth studying whether these small aggregates are still present in vivo). The relaxivity could also be further optimized by rigidifying the system and modifying the coordination sphere of $\mathrm{Gd}^{3+}$, which is the subject of future studies. All together this suggests that these bimodal systems hold great promise as MRI and NIR probes in vitro and in vivo imaging.

\section{Acknowledgments}

The Ministère de l'Enseignement Supérieur et de la Recherche, the Centre National de la Recherche Scientifique (CNRS), the Conseil Régional de Bourgogne (PhD JCE grant \# 2015-9205AAO033S04139 / BG0003203), and the French Research National Agency (ANR) via project JCJC "SPID" ANR-16-CE070020 and project JCJC "WazaBY" ANR-18-CE18-0012 are gratefully acknowledged. This work is part of the projects "Pharmacoimagerie et agents théranostiques" et "Chimie durable, environnement et agroalimentaire" supported by the Université de Bourgogne and the Conseil Régional de Bourgogne through the Plan d'Actions Régional pour l'Innovation (PARI) and the European Union through the PO FEDER-FSE Bourgogne 2014/2020 programs. It was performed within the Dijon's pharmaco-imaging consortium, a regional center of excellence in pharmacoimaging. This work was also supported by a French Government Grant managed by the French National Research Agency (ANR) under the program "Investissements d'Avenir" (reference ANR-10-EQPX-05- 01/IMAPPI Equipex). Chematech ${ }^{\circledast}$ society is warmly thanked for providing DO3A- $(\mathrm{tBu})_{3}-\mathrm{NH}_{2}$ macrocycle. FrenchBIC, GDR AIM, and OncoDesign $^{\circledast}$ are acknowledged for fruitful discussion. LAG acknowledged the French Research National Agency (ANR) for her grant (SADAM ANR-16-CE07-0015-02). Mr Soustelle, and Ms M.-J. Penouilh are gratefully acknowledged for HR-MS, NMR analyses, and Dr Myriam Laly for ionic chromatography.

\section{Supporting Information Available}

This section gathered all experimental details: synthetic procedures, analytical data $\left({ }^{1} \mathrm{H},{ }^{13} \mathrm{C},{ }^{11} \mathrm{~B},{ }^{19} \mathrm{~F}\right.$ NMR, HRMS), ${ }^{1} \mathrm{H}-\mathrm{NMR}$ spectra, analytical and semi-preparative HPLC conditions and chromatograms, ionic chromatography, protocol for determining photophysical properties, absorption, emission and excitation spectra, photophysical experiments with or without HSA and on phantoms, structures of 
the systems discussed in the article, details concerning relaxometric measurements, temperature dependent ${ }^{17} \mathrm{O}$ measurements, relaxivity measurements in the presence of HAS, phantom MRI images, full parameters used in the fitting procedure of NMRD and ${ }^{17} \mathrm{O}$ data, and equations used for the fits of ${ }^{17} \mathrm{O} N M R$ and ${ }^{1} \mathrm{H}$ NMRD data, report of singlet oxygen formation studies, and details of antiproliferative tests.

References

(1) Barabino, G.; Klein, J. P.; Porcheron, J.; Grichine, A.; Coll, J.-L.; Cottier, M. Intraoperative Near-Infrared Fluorescence Imaging Using Indocyanine Green in Colorectal Carcinomatosis Surgery: Proof of Concept. Eur J Surg Oncol 2016, 42 (12), 1931-1937. https://doi.org/10.1016/j.ejso.2016.06.389.

(2) Frangioni, J. V. In Vivo Near-Infrared Fluorescence Imaging. Curr Opin Chem Biol 2003, 7 (5), 626-634.

(3) Kiyose, K.; Aizawa, S.; Sasaki, E.; Kojima, H.; Hanaoka, K.; Terai, T.; Urano, Y.; Nagano, T. Molecular Design Strategies for Near-Infrared Ratiometric Fluorescent Probes Based on the Unique Spectral Properties of Aminocyanines. Chemistry 2009, 15 (36), 9191-9200. https://doi.org/10.1002/chem.200900035.

(4) Yuan, L.; Lin, W.; Zheng, K.; He, L.; Huang, W. Far-Red to near Infrared AnalyteResponsive Fluorescent Probes Based on Organic Fluorophore Platforms for Fluorescence Imaging. Chem Soc Rev 2013, 42 (2), 622-661. https://doi.org/10.1039/c2cs35313j.

(5) Zhu, H.; Fan, J.; Du, J.; Peng, X. Fluorescent Probes for Sensing and Imaging within Specific Cellular Organelles. Acc. Chem. Res. 2016, 49 (10), 2115-2126. https://doi.org/10.1021/acs.accounts.6b00292.

(6) Guo, Z.; Park, S.; Yoon, J.; Shin, I. Recent Progress in the Development of Near-Infrared Fluorescent Probes for Bioimaging Applications. Chem. Soc. Rev. 2014, 43 (1), 16-29. https://doi.org/10.1039/C3CS60271K.

(7) Zhao, J.; Chen, J.; Ma, S.; Liu, Q.; Huang, L.; Chen, X.; Lou, K.; Wang, W. Recent Developments in Multimodality Fluorescence Imaging Probes. Acta Pharmaceutica Sinica B 2018, 8 (3), 320-338. https://doi.org/10.1016/j.apsb.2018.03.010.

(8) James, M. L.; Gambhir, S. S. A Molecular Imaging Primer: Modalities, Imaging Agents, and Applications. Physiological Reviews 2012, 92 (2), 897-965.

https://doi.org/10.1152/physrev.00049.2010.

(9) Hernot, S.; Manen, L. van; Debie, P.; Mieog, J. S. D.; Vahrmeijer, A. L. Latest Developments in Molecular Tracers for Fluorescence Image-Guided Cancer Surgery. The Lancet Oncology 2019, 20 (7), e354-e367. https://doi.org/10.1016/S14702045(19)30317-1.

(10) Wang, Y.; Song, R.; Feng, H.; Guo, K.; Meng, Q.; Chi, H.; Zhang, R.; Zhang, Z. Visualization of Fluoride Ions In Vivo Using a Gadolinium(III)-Coumarin ComplexBased Fluorescence/MRI Dual-Modal Probe. Sensors 2016, 16 (12), 2165. https://doi.org/10.3390/s16122165.

(11) Wang, Y.; Song, R.; Guo, K.; Meng, Q.; Zhang, R.; Kong, X.; Zhang, Z. A Gadolinium(III) Complex Based Dual-Modal Probe for MRI and Fluorescence Sensing of Fluoride Ions in Aqueous Medium and in Vivo. Dalton Trans. 2016, 45 (44), 1761617623. https://doi.org/10.1039/C6DT02229D.

(12) Harrison, V. S. R.; Carney, C. E.; MacRenaris, K. W.; Waters, E. A.; Meade, T. J. Multimeric Near IR-MR Contrast Agent for Multimodal In Vivo Imaging. J. Am. Chem. Soc. 2015, 137 (28), 9108-9116. https://doi.org/10.1021/jacs.5b04509.

(13) Ceulemans, M.; Nuyts, K.; De Borggraeve, W.; Parac-Vogt, T. Gadolinium(III)-DOTA Complex Functionalized with BODIPY as a Potential Bimodal Contrast Agent for MRI 
and Optical Imaging. Inorganics 2015, 3 (4), 516-533.

https://doi.org/10.3390/inorganics3040516.

(14) Dong, D.; Jing, X.; Zhang, X.; Hu, X.; Wu, Y.; Duan, C. Gadolinium(III)-Fluorescein Complex as a Dual Modal Probe for MRI and Fluorescence Zinc Sensing. Tetrahedron 2012, 68 (1), 306-310. https://doi.org/10.1016/j.tet.2011.10.034.

(15) Yamane, T.; Hanaoka, K.; Muramatsu, Y.; Tamura, K.; Adachi, Y.; Miyashita, Y.; Hirata, Y.; Nagano, T. Method for Enhancing Cell Penetration of Gd3+-Based MRI Contrast Agents by Conjugation with Hydrophobic Fluorescent Dyes. Bioconjugate Chem. 2011, 22 (11), 2227-2236. https://doi.org/10.1021/bc200127t.

(16) You, Y.; Tomat, E.; Hwang, K.; Atanasijevic, T.; Nam, W.; Jasanoff, A. P.; Lippard, S. J. Manganese Displacement from Zinpyr-1 Allows Zinc Detection by Fluorescence Microscopy and Magnetic Resonance Imaging. Chem. Commun. 2010, 46 (23), 41394141. https://doi.org/10.1039/C0CC00179A.

(17) Huynh, A. M.; Müller, A.; Kessler, S. M.; Henrikus, S.; Hoffmann, C.; Kiemer, A. K.; Bücker, A.; Jung, G. Small BODIPY Probes for Combined Dual ${ }^{19} \mathrm{~F}$ MRI and Fluorescence Imaging. ChemMedChem 2016, 11 (14), 1568-1575. https://doi.org/10.1002/cmdc.201600120.

(18) Stasiuk, G. J.; Minuzzi, F.; Sae-Heng, M.; Rivas, C.; Juretschke, H.-P.; Piemonti, L.; Allegrini, P. R.; Laurent, D.; Duckworth, A. R.; Beeby, A.; et al. Dual-Modal Magnetic Resonance/Fluorescent Zinc Probes for Pancreatic $\beta$-Cell Mass Imaging. Chem. Eur. J. 2015, 21 (13), 5023-5033. https://doi.org/10.1002/chem.201406008.

(19) Wang, H.; Mu, X.; Yang, J.; Liang, Y.; Zhang, X.-D.; Ming, D. Brain Imaging with NearInfrared Fluorophores. Coordination Chemistry Reviews 2019, 380, 550-571. https://doi.org/10.1016/j.ccr.2018.11.003.

(20) Hong, G.; Antaris, A. L.; Dai, H. Near-Infrared Fluorophores for Biomedical Imaging. Nat Biomed Eng 2017, 1 (1), 0010. https://doi.org/10.1038/s41551-016-0010.

(21) Hilderbrand, S. A.; Weissleder, R. Near-Infrared Fluorescence: Application to in Vivo Molecular Imaging. Current Opinion in Chemical Biology 2010, 14 (1), 71-79. https://doi.org/10.1016/j.cbpa.2009.09.029.

(22) Li, J.-B.; Liu, H.-W.; Fu, T.; Wang, R.; Zhang, X.-B.; Tan, W. Recent Progress in SmallMolecule Near-IR Probes for Bioimaging. Trends in Chemistry 2019, 1 (2), 224-234. https://doi.org/10.1016/j.trechm.2019.03.002.

(23) Martinić, I.; Eliseeva, S. V.; Petoud, S. Near-Infrared Emitting Probes for Biological Imaging: Organic Fluorophores, Quantum Dots, Fluorescent Proteins, Lanthanide(III) Complexes and Nanomaterials. Journal of Luminescence 2017, 189, 19-43. https://doi.org/10.1016/j.jlumin.2016.09.058.

(24) Haque, A.; Faizi, Md. S. H.; Rather, J. A.; Khan, M. S. Next Generation NIR Fluorophores for Tumor Imaging and Fluorescence-Guided Surgery: A Review. Bioorganic \& Medicinal Chemistry 2017, 25 (7), 2017-2034. https://doi.org/10.1016/j.bmc.2017.02.061.

(25) Garland, M.; Yim, J. J.; Bogyo, M. A Bright Future for Precision Medicine: Advances in Fluorescent Chemical Probe Design and Their Clinical Application. Cell Chemical Biology 2016, 23 (1), 122-136. https://doi.org/10.1016/j.chembiol.2015.12.003.

(26) Ge, Y.; O’Shea, D. F. Azadipyrromethenes: From Traditional Dye Chemistry to Leading Edge Applications. Chem. Soc. Rev. 2016, 45 (14), 3846-3864. https://doi.org/10.1039/C6CS00200E.

(27) Pliquett, J.; Dubois, A.; Racoeur, C.; Mabrouk, N.; Amor, S.; Lescure, R.; Bettaïeb, A.; Collin, B.; Bernhard, C.; Denat, F.; et al. A Promising Family of Fluorescent WaterSoluble Aza-BODIPY Dyes for in Vivo Molecular Imaging. Bioconjugate Chem. 2019, 30 (4), 1061-1066. https://doi.org/10.1021/acs.bioconjchem.8b00795. 
(28) Kim, E.-J.; Bhuniya, S.; Lee, H.; Kim, H. M.; Shin, W. S.; Kim, J. S.; Hong, K. S. In Vivo Tracking of Phagocytic Immune Cells Using a Dual Imaging Probe with Gadolinium-Enhanced MRI and Near-Infrared Fluorescence. ACS Appl. Mater. Interfaces 2016, 8 (16), 10266-10273. https://doi.org/10.1021/acsami.6b03344.

(29) Fredy, J. W.; Scelle, J.; Guenet, A.; Morel, E.; Adam de Beaumais, S.; Ménand, M.; Marvaud, V.; Bonnet, C. S.; Tóth, E.; Sollogoub, M.; et al. Cyclodextrin Polyrotaxanes as a Highly Modular Platform for the Development of Imaging Agents. Chemistry - A European Journal 2014, 20 (35), 10915-10920. https://doi.org/10.1002/chem.201403635.

(30) Wu, D.; Daly, H. C.; Conroy, E.; Li, B.; Gallagher, W. M.; Cahill, R. A.; O’Shea, D. F. PEGylated BF2-Azadipyrromethene (NIR-AZA) Fluorophores, for Intraoperative Imaging. European Journal of Medicinal Chemistry 2019, 161, 343-353. https://doi.org/10.1016/j.ejmech.2018.10.046.

(31) Yang, Z.; Bai, X.; Ma, S.; Liu, X.; Zhao, S.; Yang, Z. A Benzoxazole Functionalized Fluorescent Probe for Selective $\mathrm{Fe}^{3+}$ Detection and Intracellular Imaging in Living Cells. Anal. Methods 2017, 9 (1), 18-22. https://doi.org/10.1039/C6AY02660E.

(32) Bodio, E.; Denat, F.; Goze, C. BODIPYS and Aza-BODIPYS Derivatives as Promising Fluorophores for in Vivo Molecular Imaging and Theranostic Applications. $J$. Porphyrins Phthalocyanines 2019, S1088424619501268. https://doi.org/10.1142/S1088424619501268.

(33) Daly, H. C.; Sampedro, G.; Bon, C.; Wu, D.; Ismail, G.; Cahill, R. A.; O’Shea, D. F. BF 2 -Azadipyrromethene NIR-Emissive Fluorophores with Research and Clinical Potential. European Journal of Medicinal Chemistry 2017, 135, 392-400. https://doi.org/10.1016/j.ejmech.2017.04.051.

(34) Grossi, M.; Morgunova, M.; Cheung, S.; Scholz, D.; Conroy, E.; Terrile, M.; Panarella, A.; Simpson, J. C.; Gallagher, W. M.; O’Shea, D. F. Lysosome Triggered Near-Infrared Fluorescence Imaging of Cellular Trafficking Processes in Real Time. Nat Commun 2016, 7 (1), 10855. https://doi.org/10.1038/ncomms10855.

(35) Bellier, Q.; Pégaz, S.; Aronica, C.; Le Guennic, B.; Andraud, C.; Maury, O. NearInfrared Nitrofluorene Substitued Aza-Boron-Dipyrromethenes Dyes. Org. Lett. 2011, 13 (1), 22-25. https://doi.org/10.1021/ol102701v.

(36) Brücher, E.; Tircsó, G.; Baranyai, Z.; Kovács, Z.; Sherry, A. D. Stability and Toxicity of Contrast Agents. In The Chemistry of Contrast Agents in Medical Magnetic Resonance Imaging; John Wiley \& Sons, Ltd, 2013; pp 157-208. https://doi.org/10.1002/9781118503652.ch4.

(37) Wahsner, J.; Gale, E. M.; Rodríguez-Rodríguez, A.; Caravan, P. Chemistry of MRI Contrast Agents: Current Challenges and New Frontiers. Chem. Rev. 2019, 119 (2), 9571057. https://doi.org/10.1021/acs.chemrev.8b00363.

(38) Loudet, A.; Bandichhor, R.; Wu, L.; Burgess, K. Functionalized BF2 Chelated Azadipyrromethene Dyes. Tetrahedron 2008, 64 (17), 3642-3654. https://doi.org/10.1016/j.tet.2008.01.117.

(39) Jing, X.; Yu, F.; Chen, L. Visualization of Nitroxyl (HNO) in Vivo via a LysosomeTargetable near-Infrared Fluorescent Probe. Chem. Commun. 2014, 50 (91), 1425314256. https://doi.org/10.1039/C4CC07561G.

(40) Bhuniya, S.; Lee, M. H.; Jeon, H. M.; Han, J. H.; Lee, J. H.; Park, N.; Maiti, S.; Kang, C.; Kim, J. S. A Fluorescence off-on Reporter for Real Time Monitoring of Gemcitabine Delivery to the Cancer Cells. Chem. Commun. 2013, 49 (64), 7141-7143. https://doi.org/10.1039/C3CC42653J.

(41) Kamkaew, A.; Burgess, K. Aza-BODIPY Dyes with Enhanced Hydrophilicity. Chem. Commun. 2015, 51 (53), 10664-10667. https://doi.org/10.1039/C5CC03649F. 
(42) Daly, H. C.; Sampedro, G.; Bon, C.; Wu, D.; Ismail, G.; Cahill, R. A.; O’Shea, D. F. BF2-Azadipyrromethene NIR-Emissive Fluorophores with Research and Clinical Potential. Eur J Med Chem 2017, 135, 392-400. https://doi.org/10.1016/j.ejmech.2017.04.051.

(43) Brouwer, A. M. Standards for Photoluminescence Quantum Yield Measurements in Solution (IUPAC Technical Report). Pure and Applied Chemistry 2011, 83 (12), 22132228. https://doi.org/10.1351/PAC-REP-10-09-31.

(44) Karatay, A.; Miser, M. C.; Cui, X.; Küçüköz, B.; Yılmaz, H.; Sevinç, G.; Akhüseyin, E.; Wu, X.; Hayvali, M.; Yaglioglu, H. G.; et al. The Effect of Heavy Atom to Two Photon Absorption Properties and Intersystem Crossing Mechanism in Aza-BoronDipyrromethene Compounds. Dyes and Pigments 2015, 122, 286-294. https://doi.org/10.1016/j.dyepig.2015.07.002.

(45) Singlet Oxygen; 2016; Vol. 1. https://doi.org/10.1039/9781782622208.

(46) Schmitt, J.; Heitz, V.; Sour, A.; Bolze, F.; Kessler, P.; Flamigni, L.; Ventura, B.; Bonnet, C. S.; Tóth, É. A Theranostic Agent Combining a Two-Photon-Absorbing Photosensitizer for Photodynamic Therapy and a Gadolinium(III) Complex for MRI Detection. Chemistry - A European Journal 2016, 22 (8), 2775-2786. https://doi.org/10.1002/chem.201503433.

(47) Carné-Sánchez, A.; Bonnet, C. S.; Imaz, I.; Lorenzo, J.; Tóth, É.; Maspoch, D. Relaxometry Studies of a Highly Stable Nanoscale Metal-Organic Framework Made of $\mathrm{Cu}(\mathrm{II}), \mathrm{Gd}(\mathrm{III})$, and the Macrocyclic DOTP. J. Am. Chem. Soc. 2013, 135 (47), 1771117714. https://doi.org/10.1021/ja4094378.

(48) Nicolle, G. M.; Tóth, É.; Eisenwiener, K.-P.; Mäcke, H. R.; Merbach, A. E. From Monomers to Micelles: Investigation of the Parameters Influencing Proton Relaxivity. $J$ Biol Inorg Chem 2002, 7 (7), 757-769. https://doi.org/10.1007/s00775-002-0353-3.

(49) Martins, A. F.; Morfin, J.-F.; Geraldes, C. F. G. C.; Tóth, É. Gd3+ Complexes Conjugated to Pittsburgh Compound B: Potential MRI Markers of $\beta$-Amyloid Plaques. $J$ Biol Inorg Chem 2014, 19 (2), 281-295. https://doi.org/10.1007/s00775-013-1055-8.

(50) Isaac, M.; Pallier, A.; Szeremeta, F.; Bayle, P.-A.; Barantin, L.; Bonnet, C. S.; Sénèque, O. MRI and Luminescence Detection of Zn2+ with a Lanthanide Complex-Zinc Finger Peptide Conjugate. Chem. Commun. 2018, 54 (53), 7350-7353. https://doi.org/10.1039/C8CC04366C.

(51) The Chemistry of Contrast Agents in Medical Magnetic Resonance Imaging: Helm/The Chemistry of Contrast Agents in Medical Magnetic Resonance Imaging; Merbach, A., Helm, L., Tóth, É., Eds.; John Wiley \& Sons, Ltd: Chichester, UK, 2013; Vol. Ch.2, p.25. https://doi.org/10.1002/9781118503652.

(52) Raiford, D. S.; Fisk, C. L.; Becker, E. D. Calibration of Methanol and Ethylene Glycol Nuclear Magnetic Resonance Thermometers. Anal. Chem. 1979, 51 (12), 2050-2051. https://doi.org/10.1021/ac50048a040.

(53) Meiboom, S.; Gill, D. Modified Spin-Echo Method for Measuring Nuclear Relaxation Times. Review of Scientific Instruments 1958, 29 (8), 688-691. https://doi.org/10.1063/1.1716296.

(54) Micskei, K.; Helm, L.; Brucher, E.; Merbach, A. E. Oxygen-17 NMR Study of Water Exchange on Gadolinium Polyaminopolyacetates [Gd(DTPA)(H2O)]2- and [Gd(DOTA) (H2O)]- Related to NMR Imaging. Inorg. Chem. 1993, 32 (18), 3844-3850. https://doi.org/10.1021/ic00070a013.

(55) Hugi, A. D.; Helm, L.; Merbach, A. E. Water Exchange on Hexaaquavanadium(III): a Variable-Temperature and Variable-Pressure17O-NMR Study at 1.4 and 4.7 Tesla. Helv. Chim. Acta 1985, 68 (2), 508-521. https://doi.org/10.1002/hlca.19850680224. 

TOC Graphic

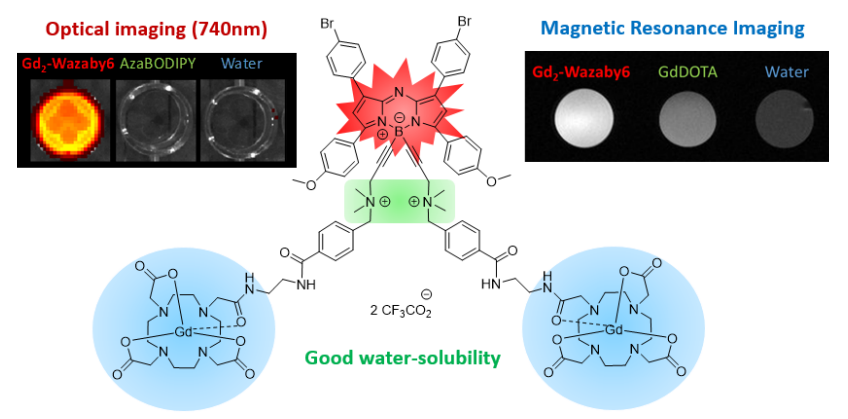

Synthesis and characterization of an original aza-BODIPY system comprising two $\mathrm{Gd}^{3+}$ complexes for magnetic resonance imaging (MRI)/optical imaging (OI) application, by functionalization of the boron center. This is the first aza-BODIPY based bimodal probe displaying a high brightness in near infrared fluorescence $\left(\lambda_{\mathrm{em}}=740 \mathrm{~nm}\right)$, an excellent relaxivity for MRI, and displaying good water solubility. The novel bimodal probe possesses all the requirements for in vivo application. 


\section{Aza-BODIPY Platform: Towards an Efficient Water-Soluble Bimodal Imaging Probe for MRI and Near-Infrared Fluorescence}

Océane Florès ${ }^{a}$, Jacques Pliquett ${ }^{a}$, Laura Abad-Galan ${ }^{b}$, Robin Lescure ${ }^{a}$, Franck Denat ${ }^{a}$, Olivier Maury ${ }^{b}$, Agnès Pallier ${ }^{c}$, Pierre-Simon Bellaye ${ }^{d}$, Bertrand Collin ${ }^{a-d}$, Sandra Même ${ }^{c}$, Célia S. Bonnet ${ }^{c *}$, Ewen Bodio $^{\text {a* }}$, Christine Goze ${ }^{\text {a* }}$.

[a] CNRS, Université Bourgogne Franche-Comté, ICMUB UMR6302 - CNRS, F-21000 Dijon, France. [b] Univ Lyon, Ecole Normale Supérieure de Lyon, CNRS, Université Claude Bernard Lyon 1, Laboratoire de Chimie, UMR 5182, F-69342 Lyon, France.

[c] Centre de Biophysique Moléculaire, CNRS, Université d’Orléans, Rue Charles Sadron, 45071 Orléans Cedex 2, France.

[d] Centre Georges François Leclerc, Service de médecine nucléaire (plateforme d'imagerie et de radiothérapie précliniques), 1 rue Professeur Marion, BP77980, 21079 Dijon Cedex

\section{Abstract}

In this study, an original aza-BODIPY system comprising two $\mathrm{Gd}^{3+}$ complexes has been designed and synthesized for magnetic resonance imaging/optical imaging application, by functionalization of the boron center. This strategy enabled to obtain a positively-charged bimodal probe, which displays an increased water-solubility, optimized photophysical properties in the near-infrared region, and very promising relaxometric properties. The absorption and emission wavelengths are 705 and $741 \mathrm{~nm}$ respectively, with a quantum yield of around $10 \%$ in aqueous media. Moreover, the system does not produce singlet oxygen upon excitation, which would be toxic for tissues. The relaxivity obtained is high at intermediate fields $\left(16.1 \mathrm{mM}^{-1} \cdot \mathrm{s}^{-1}\right.$ at $20 \mathrm{MHz}$ and $\left.310 \mathrm{~K}\right)$ and competes with that of bigger or more rigid systems. A full relaxometric and ${ }^{17} \mathrm{O} N M R$ study and fitting of the data using the Lipari-Szabo approach showed that this high relaxivity can be explained by the size of the system and the presence of some small aggregates. These optimized photophysical and relaxometric properties highlight the potential use of such systems for future bimodal imaging studies.

\section{Introduction}

Imaging and especially molecular imaging has gained an increasing interest during these last years. Indeed, the insights in this field facilitate an earlier and more reliable diagnostic, enable the monitoring of the efficiency of a therapy, and even assist the surgeon during operations. ${ }^{1-6}$ Therefore, molecular imaging can benefit to a large number of pathologies. Moreover, this imaging approach also permits the discovery of novel biological targets or biomarkers and help to understand mechanisms of different diseases. Each molecular imaging technique has its own advantages and drawbacks and synergy is expected by association of two complementary modalities in a single molecular probe. ${ }^{7}$ This is particularly true when combining the two non-ionizing techniques: Magnetic Resonance Imaging (MRI) with Optical Imaging (OI). Indeed, MRI has an excellent spatial resolution and offers 3D anatomical and also functional information, which is ideal for in vivo imaging, but it suffers from low sensitivity. On the other hand, Ol exhibits high sensitivity and high microscopic resolution $(<10 \mathrm{~nm}$ in the case of super resolved fluorescent microscopy), but suffers from poor tissue penetration. ${ }^{8}$ Another advantage of OI is that it enables real time imaging. Moreover, more recently, despite its limited penetration depth, it has gained an increasing interest in the clinic for intra-operative fluorescence assisted surgery, where the use of fluorescence imaging particularly helps the surgeon to better delineate tumor margins, identify the lymph nodes invaded by cancer cells... ${ }^{9}$ 
In order to perform bimodal MRI/OI, there is an increasing interest in the conception of new efficient multimodal probes. Indeed, combination of these two imaging modalities into a single molecule assures the identical biodistribution compared to the combination of two separate imaging agents, and thus enables to perform a much more accurate diagnosis. The design of such bimodal probes is a real challenge, mainly because of the difference of sensitivity between MRI and OI. Therefore, the majority of the reported MRI/OI agents are based on functionalized polymers or nanoparticles. However small molecular bimodal agents were also reported and proved to be very effective. These agents combine fluorine atoms for ${ }^{19} \mathrm{~F}$ MRI or one, two, or more complexes, which are active in MRI, such as $\mathrm{Mn}^{2+}$ or $\mathrm{Gd}^{3+}$ complexes, and one fluorophore, which can be a dipyridophenazine, fluorescein, cyanine, rhodamine, quinoline, BODIPY... ${ }^{10-18}$

For in vivo optical imaging applications, the fluorophores need to absorb and emit in the spectral range, where biological tissues are more transparent and less scattering. This region, often called biological, transparency or theranostic window, corresponds to near infrared (NIR, as example NIR I corresponds to $650-900 \mathrm{~nm}$ ). ${ }^{19-21}$ Development of NIR fluorophores is a hot topic because, despite the diversity of reported fluorophores, it seems that none of them is ideal for in vivo applications, and that most of them lack of chemical and photochemical stability, are poorly water soluble, low emissive, difficult to functionalize and their synthesis is time-consuming. ${ }^{22-25}$ Very recently, the aza-BODIPY family attracted considerable attention because it may be one of the best candidates to tackle all these issues. These very stable BODIPY derivatives display a lower brightness than well-known NIR cyanines such as ICG and IRDye ${ }^{\circledR} 800 \mathrm{CW}$ (Figure 1), but can be rapidly synthesized in large scale and are easily functionalized. ${ }^{26}$
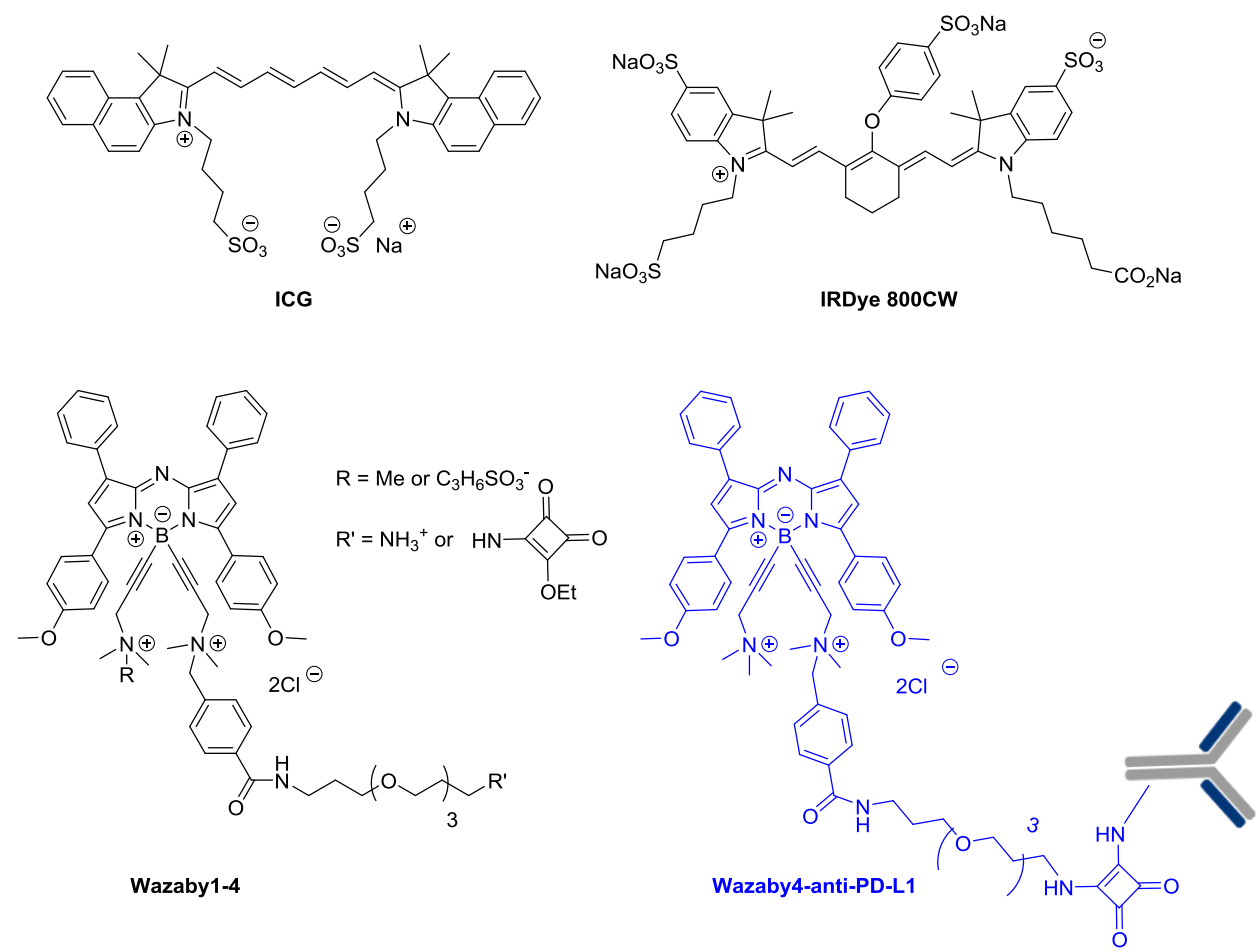

Figure 1: structure of some optical imaging clinically used contrast agents and of Wazaby (watersoluble aza-BODIPY), we reported previously ${ }^{27}$

However, despite their potential, there are only very scarce reports of bimodal MRI/OI probes including (aza)-BODIPY. They have been used in combination with several fluorine atoms for ${ }^{19} \mathrm{~F} \mathrm{MRI},{ }^{17}$ or with two GdDTPA complexes, ${ }^{28}$ or GdDOTA complexes in a monomolecular system ${ }^{13}$ or in a polyrotaxane ${ }^{29}$. The sensitivity of ${ }^{19} \mathrm{~F}$ MRI remains lower than that of ${ }^{1} \mathrm{H}$ MRI. GdDTPA complexes are not the best candidates for in vivo use (vide infra) and the relaxivity of the BODIPY-GdDOTA system (vide infra) can certainly be optimized. This leaves room for improvement of such systems. It should 
also be noted that a bisGdDTPA-aza-BODIPY system was recently reported by Kim, Long and coll. to track in vivo phagocytic immune cells by MRI/OI bimodal imaging, highlighting the potential of such systems. ${ }^{28}$

Despite their interesting properties, the limited use of BODIPY in bimodal imaging can certainly be explained by their poor water solubility, which is problematic for their use in vivo, and particularly for MRI. ${ }^{30-32}$ Several groups tethered water-solubilizing groups to the aza-BODIPY core, but even if sometimes it enabled in vitro and in vivo investigations, the resulting probes were still subject to significant aggregation phenomena (requiring the addition of surfactant). ${ }^{30,33,34}$ Very recently, some of us succeeded in designing fully water-soluble aza-BODIPY derivatives, called Wazaby (for water-soluble aza-BODIPY), thanks to the introduction of ammonium groups via boron functionalization (functionalizing boron induced aza-BODIPY faces hindrance, which prevented aggregation) (Figure 1). ${ }^{27}$ These probes were bioconjugated onto antibodies, were very stable in physiological media, and presented better in vivo optical properties than the Cy5, used as a control.

Building on this success, we decided to further investigate the potential of these Wazaby dyes and to conceive MRI/OI MOnomolecular Multimodal Imaging Probes (MOMIP). For this aim, we decided to attach two gadolinium complexes onto the boron-functionalized arms of the Wazaby scaffold, thanks to the strategy developed previously (Figure 2). Concerning the aza-BODIPY core, we chose an azaBODIPY bearing methoxy groups on the aromatic rings at the "south" of the molecule (also called proximal phenyl groups in aza-BODIPY chemistry) in order to shift the maximal absorption wavelength around $690-700 \mathrm{~nm}$. To maximize this effect (maximal absorption wavelength $>700 \mathrm{~nm}$ ) by creating a greater "push-pull" effect, bromine atoms were introduced on the aromatic rings at the "north" of the molecule (distal phenyl groups). It has to be noted that this functional group would enable further functionalization of the molecule in the future (extension of conjugation or introduction of a bioconjugatable handle if vectorization is needed). ${ }^{35}$

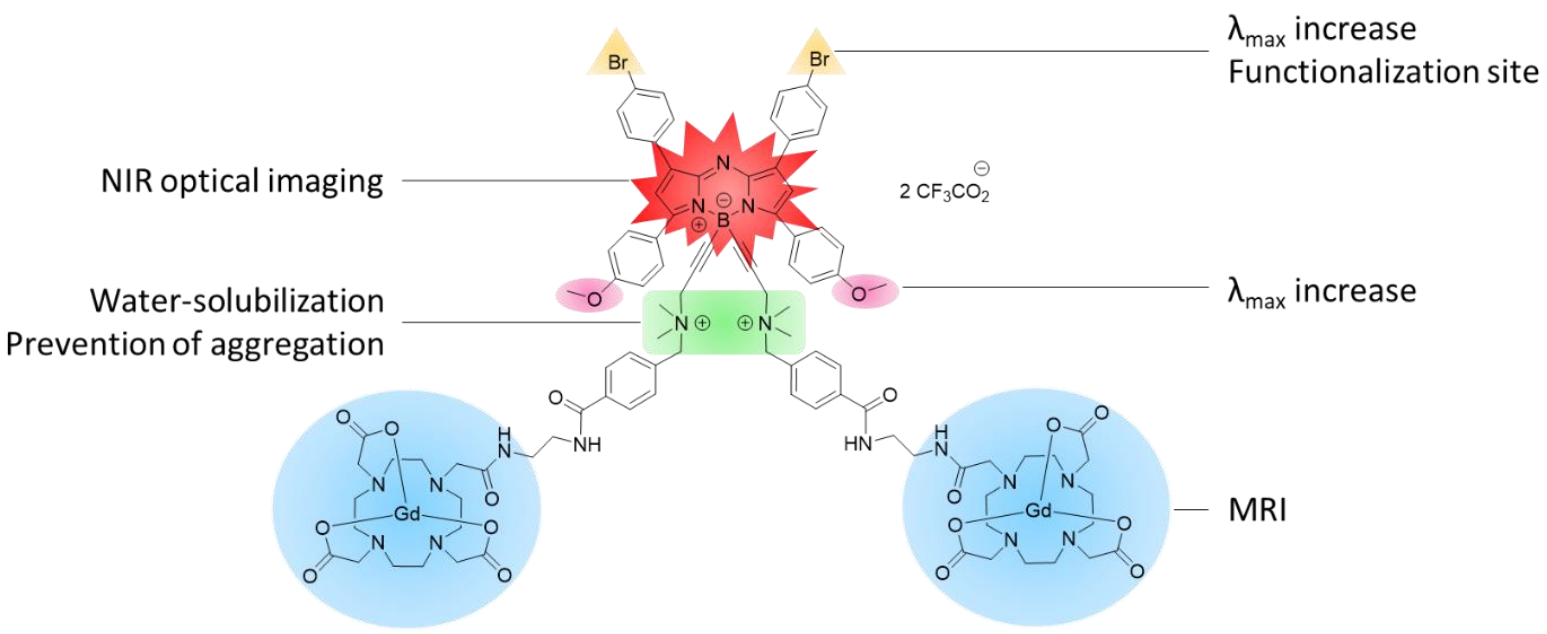

Figure 2 : Targeted bimodal probe and justification of the chosen functionalization

In MRI, DOTAREM ${ }^{\circledR}$ is one of the most clinically used contrast agents due to its high thermodynamic stability and kinetic inertness, which is essential for the safe use of the probe. ${ }^{36}$ Indeed, free gadolinium ions are highly toxic, and recently, complexes with lower kinetic inertness have been shown to increase the risk of nephrogenic systemic fibrosis (NSF), especially for patients having renal failure. ${ }^{37}$ As macrocyclic chelates are known to be more stable than linear ones, a DOTA derivative (DOTA monoamide or DOTAMA) was used for $\mathrm{Gd}^{3+}$ complexation. 
Aza-BODIPY 2 was synthesized at the gram scale according to the method we previously reported, ${ }^{27}$ starting by the synthesis of the aza-BODIPY $1,{ }^{38}$ followed by the substitution of the fluorine atoms on the boron center using the Grignard reagent of $N, N$-dimethylpropargylamine. The resulting intermediate platform was then alkylated by an excess of 4-(bromomethyl)benzoic acid in presence of $\mathrm{NaHCO}_{3}$ (Scheme 1) to give Wazaby5. Special attention was paid to the base employed as proton scavenger. A stronger base led to the formation of small amount of ester side product - alkylation of the carboxylate -, leading to a tricky purification.
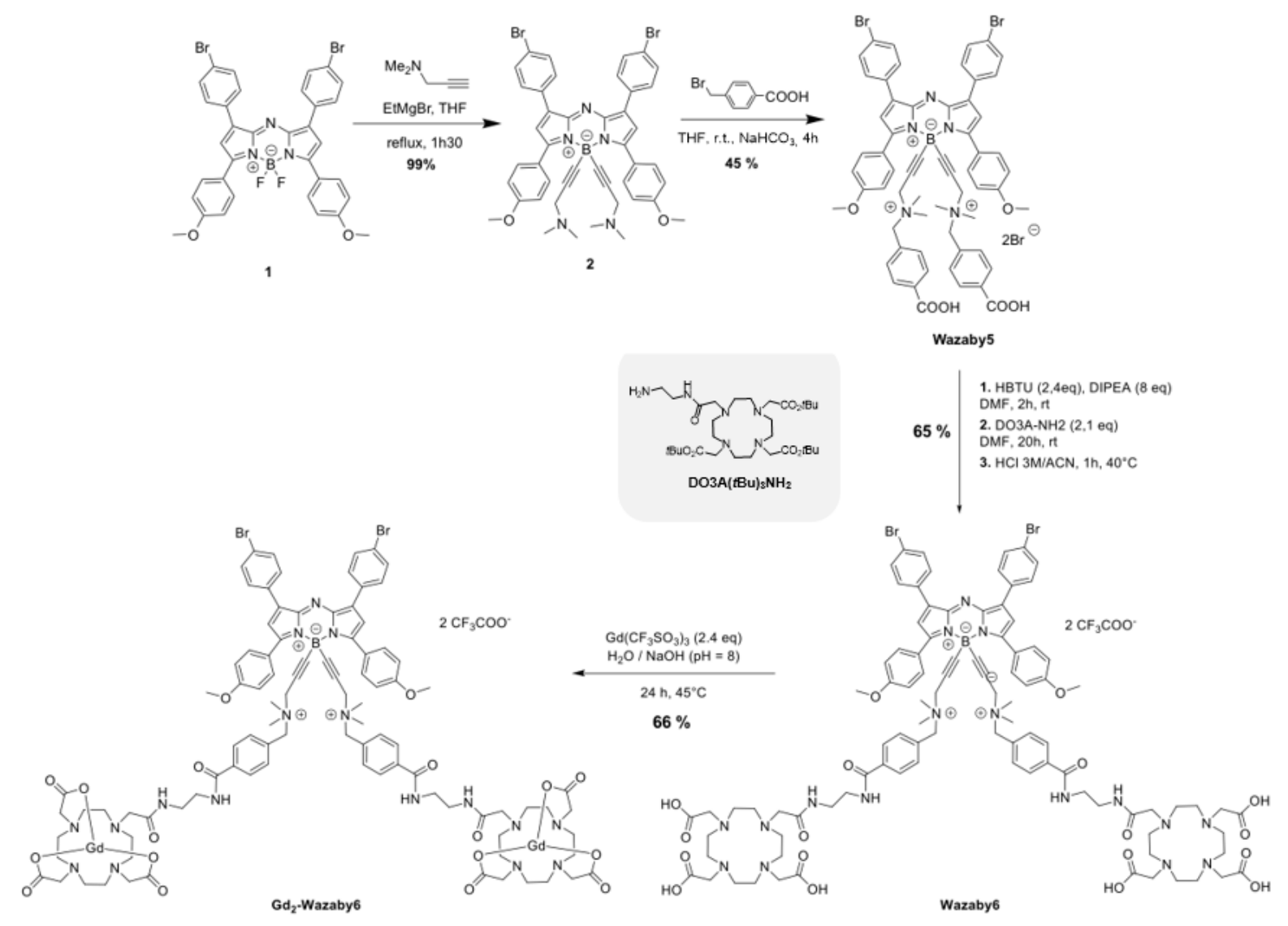

Scheme 1: synthetic route toward the bimodal probe $\mathrm{Gd}_{2}$-Wazaby6

Wazaby5 was reacted with a macrocyclic derivative $\left(\mathrm{DO} 3 \mathrm{~A}(t \mathrm{Bu})_{3} \mathrm{NH}_{2}\right)$ in classic peptidic coupling reaction conditions and directly treated by $\mathrm{HCl} 3 \mathrm{M}$ in order to hydrolyze the protected tertiobutylester arms. The bis-DOTA derivative was then purified by semi-preparative HPLC to lead to the formation of pure Wazaby6. It is worth noting that the fluxionality of macrocycles implied to record NMR spectra at high temperature. A representation of variable temperature ${ }^{1} \mathrm{H}-\mathrm{NMR}$ of Wazaby6 is displayed in Figure S2.

Gadolinium was introduced into the two macrocycle cavities by reacting 2.4 eq of $\mathrm{Gd}\left(\mathrm{CF}_{3} \mathrm{SO}_{3}\right)_{3}$ salt with Wazaby6 in water in presence of sodium hydroxyde. The resulting homobimetallic complex $\mathbf{G d}_{2}$ Wazaby6 was characterized by high resolution mass spectrometry (HRMS), HPLC, elemental analysis and ionic chromatography but not NMR due to its paramagnetic character. 
As highlighted in the introduction, aza-BODIPY fluorophores display very interesting properties for in vivo optical imaging. However, in general, their main drawback is their poor solubility and their tendency to aggregate in water and in physiological media, despite the introduction of solubilizing groups. Most of the time, imaging experiments are performed in the presence of surfactants like Cremophor EL or castor oil. ${ }^{39-42}$ Therefore, it was crucial for us to investigate the photophysical properties of our bimodal compounds in organic solvent (DMF, DMSO), as well as in PBS (PhosphateBuffered Saline), without adding any surfactant.

Table 1: photophysical data of Wazaby5, Wazaby6, and $\mathbf{G d}_{\mathbf{2}}$-Wazaby6 in different solvents at $298 \mathrm{~K}$ at $2 \mu M\left(\lambda_{\text {exc }}=670 \mathrm{~nm}\right)$.

\begin{tabular}{|c|c|c|c|c|c|}
\hline Compound & Solvent & $\begin{array}{c}\lambda_{\max , \mathrm{Abs}} \\
{[\mathrm{nm}]}\end{array}$ & $\begin{array}{c}\varepsilon_{\lambda \max } \\
{\left[\mathrm{M}^{-1} \mathrm{~cm}^{-1}\right]}\end{array}$ & $\begin{array}{c}\lambda_{\max , \mathrm{Em}} \\
{[\mathrm{nm}]}\end{array}$ & $\phi_{\mathrm{F}}[\%]^{*}$ \\
\hline \multirow{2}{*}{ Wazaby5 } & DMSO & 704 & 74,000 & 739 & 29 \\
\hline \multirow{3}{*}{ Gd $_{2}$-Wazaby6 } & DMSO & 705 & 82,000 & 741 & 15 \\
\cline { 2 - 8 } & PBS & $\mathbf{7 0 5}$ & $\mathbf{4 6 , 0 0 0}$ & $\mathbf{7 4 1}$ & $\mathbf{1 0}$ \\
& DMSO & 705 & 78,000 & 739 & 22 \\
\cline { 2 - 8 } & PBS & $\mathbf{7 0 5}$ & $\mathbf{4 1 , 0 0 0}$ & $\mathbf{7 4 1}$ & $\mathbf{9}$ \\
\cline { 2 - 7 } & water & 705 & 49,000 & 741 & 10 \\
\hline
\end{tabular}

* reference: aza-BODIPY $3\left(\phi_{\mathrm{F}}=36 \% \text { in chloroform, } 298 \mathrm{~K}, \lambda_{\mathrm{exc}}=670 \mathrm{~nm}\right)^{43}$

The photophysical data of Wazaby5, Wazaby6, and the related bimetallic complex $\mathbf{G d}_{2}$-Wazaby6 were studied in DMSO, DMF, and PBS, and results are depicted in Table 1, Figure 3, and Table S2. The three compounds display comparable absorption and emission profiles, with absorption wavelength at $\approx 705$ $\mathrm{nm}$ and emission maxima at $\approx 740 \mathrm{~nm}$. Interestingly, the introduction of the two macrocycles as well as the complexation of the two gadolinium atoms had almost no impact on the photophysical properties of the fluorophore.

When comparing Wazaby6 and $\mathbf{G d}_{2}$-Wazaby6 in DMSO and PBS, we can notice that the absorption, emission and excitation profiles of the compounds are comparable in both media (Figure 3), even if we can observe that absorption band is slightly larger than excitation one between 600 and $650 \mathrm{~nm}$ in PBS. It suggests that very limited aggregation occurs in PBS. If these spectra are compared to the ones of Kim's and Long's system, ${ }^{28}$ it clearly highlights the interest of boron-functionalization for limiting aggregation phenomenon, which is really frequent for aza-BODIPY fluorophores. Interestingly, the quantum yield efficiency remains comparable both in organic solvents (around 20\%) and in PBS (around 10\%). Such values are good for NIR emitters and are clearly enough to undertake future OI experiments.

It is well known that the presence of "heavy atoms" induces an increase of the spin-orbit coupling and therefore promotes the generation of singlet oxygen. ${ }^{32,44}$ However, this effect is generally more substantial when the heavy atoms occupy a position in the core region of the aza-BODIPY. In the present study, a non-toxic probe is under scope, thus, the production of singlet oxygen needs to be controlled. In order to test if toxic singlet oxygen is generated, the probes were dissolved in a nonprotic solvent (DMF) for feasible observation of the phosphorescence band of singlet oxygen at $1270 \mathrm{~nm}$, as protic solvents will efficiently quench its emission. ${ }^{45}$ In every case this band was found to be absent, which confirms that neither Wazaby6 nor $\mathbf{G d}_{2}$-Wazaby 6 produce singlet oxygen, when 
irradiated (see supporting information for details). Moreover, preliminary antiproliferative tests on HepG2 cells (Human hepatocellular carcinoma, ATCC ${ }^{\circ}$ HB-8065 $5^{\text {TM }}$ ) confirmed that Gd $_{2}$-Wazaby6 do not display significant toxicity $\left(\mathrm{IC}_{50}\right.$ at $\left.24 \mathrm{~h}>100 \mu \mathrm{M}\right)$. Additional experiments will be performed in the future to ascertain this aspect.

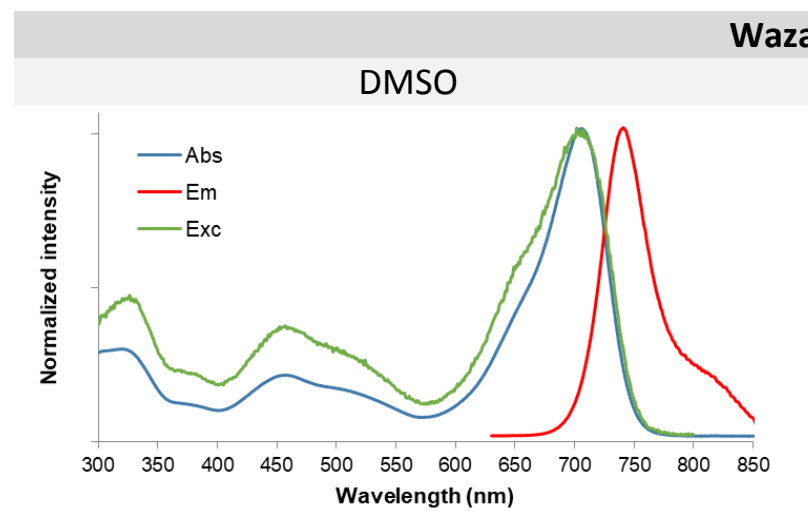

Wazaby6

PBS

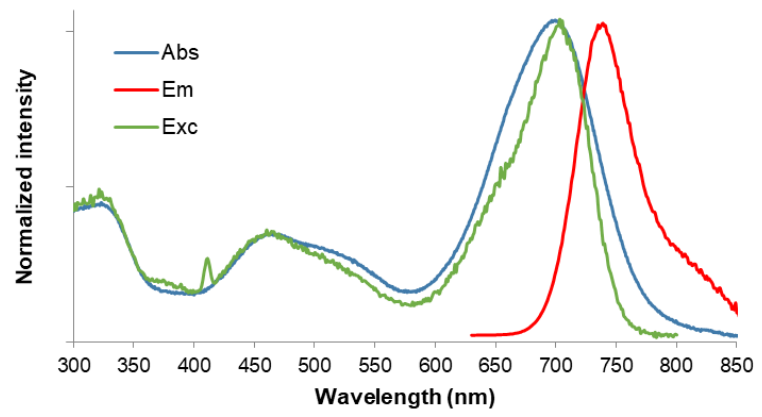

$\mathrm{Gd}_{2}$-Wazaby6

DMSO
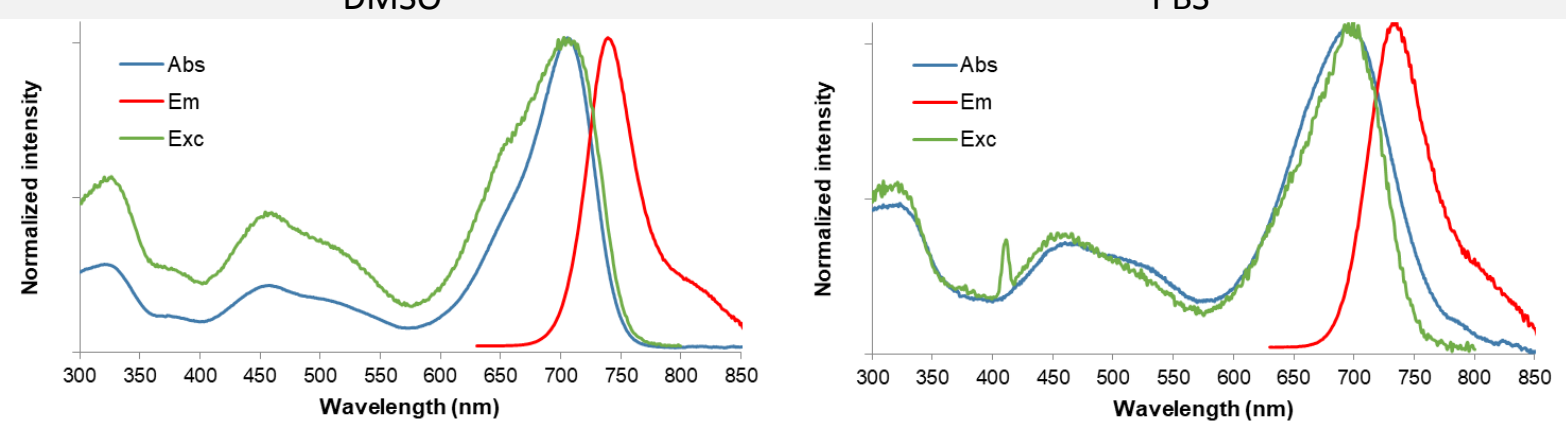

Figure 3: Normalized absorption, emission, and excitation spectra of Wazaby6 and $\mathbf{G d}_{2}$-Wazaby6 at $298 \mathrm{~K}\left(\lambda_{\text {ex }}=620 \mathrm{~nm}\right.$ for emission spectra, detection at $\lambda_{e m}=820 \mathrm{~nm}$ for excitation spectra)

Relaxivity studies

Given the good solubility of the system, relaxivity measurements could be undertaken to characterize the efficiency of the system as an MRI probe. To this aim, NMRD (Nuclear Magnetic Resonance Dispersion) profiles were measured in water at $0.64 \mathrm{mM}$ at $\mathrm{pH}=7.2$ in the field range $40 \mathrm{kHz}-400$ $\mathrm{MHz}$ at two different temperatures $(298 \mathrm{~K}$ and $310 \mathrm{~K}$ ) as illustrated in Figure 4.

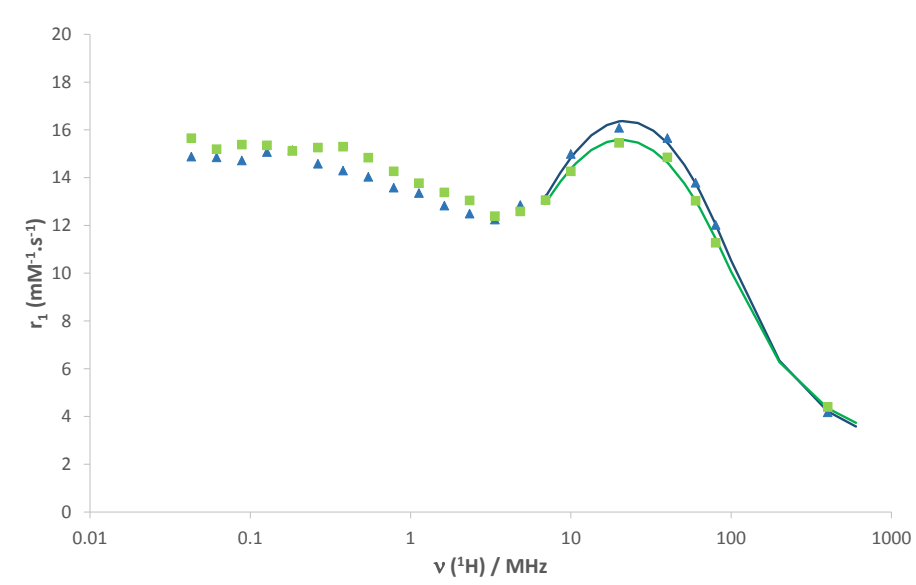

Figure 4: ${ }^{1} \mathrm{H}$ NMRD profile of $\mathrm{Gd}_{2}$-Wazaby6 $0.64 \mathrm{mM}$ in water, $\mathrm{pH}=7.2$ at $298 \mathrm{~K}(\square)$ and $310 \mathrm{~K}(\Delta)$. The lines represent the best fit to the SBM theory. 
Interestingly, the temperature dependence of the system shows that we are in an intermediate regime where the rotation of the system starts to limit relaxivity rather than the water exchange rate. Indeed, the $37^{\circ} \mathrm{C}$ relaxivities are very close and even higher than those at $25^{\circ} \mathrm{C}$. Typically, when the rotation is the limiting parameter (as observed for small molecular systems), relaxivity is decreasing when increasing temperature, whereas when the water exchange rate is limiting, relaxivity is increasing with increasing temperature. This result is also in accordance with the characteristic hump of slowlyrotating species visible on the NMRD profiles at intermediate fields $(20-80 \mathrm{MHz})$. As this system remains quite flexible, we decided to investigate the relaxivity as a function of the $\mathrm{Gd}^{3+}$ concentration. Relaxivities were measured in the $\mathrm{Gd}^{3+}$ concentration range $0.1-4 \mathrm{mM}(0.05-2 \mathrm{mM}$ for the system) both in water at $\mathrm{pH}=7.2$ and in $\mathrm{PBS}$ at $\mathrm{pH}=7.4$, and the results are presented in Figure 5. At the concentration used for measuring relaxivities ( $0.64 \mathrm{mM}$ of $\mathbf{G d}_{2}$-Wazaby6), we can see that some aggregation occurs both in water and PBS probably due to interactions between the aza-BODIPY units. This is in accordance with the photophysical studies, highlighting small aggregation, but which doesn't prevent water solubility. The aggregation is favored in PBS, which can be explained by additional electrostatic interactions between the $\mathbf{G d}_{2}$-Wazaby $\mathbf{6}$ cation (positive charge) and PBS (minus charge). This phenomenon certainly explains the temperature dependency of this otherwise quite flexible system.

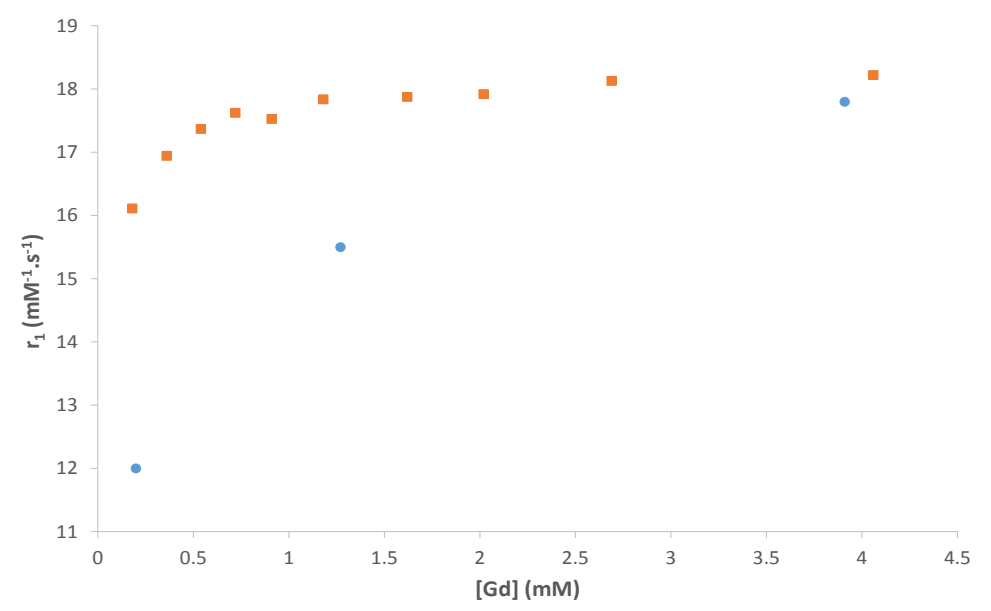

Figure 5: Relaxivity of the complex as a function of $\mathrm{Gd}^{3+}$ concentration at $298 \mathrm{~K}, 20 \mathrm{MHz}$, in water at $p H=7.2(\bullet)$ and in PBS at $p H=7.4(\square)$.

The relaxivity of the system at $0.64 \mathrm{mM}$ in water and at $20 \mathrm{MHz}, 310 \mathrm{~K}$ is $16.1 \mathrm{mM}^{-1} . \mathrm{s}^{-1}$. As a comparison, and as expected due to the aggregation phenomenon, it is considerably higher than that of the mononuclear GdDOTA-BODIPY complex $\left(3.9 \mathrm{mM}^{-1} \cdot \mathrm{s}^{-1}\right)^{13}$ and the relaxivity of the Bis-GdDTPA-azaBODIPY system is $13.3 \mathrm{mM}^{-1} \cdot \mathrm{s}^{-1}$ at $60 \mathrm{MHz}$ and room temperature ${ }^{28}$ (see Figure S10 for structures). The relaxivity is in the same order of magnitude than a multimodal polyrotaxane comprising cyclodextrins bearing GdDOTAMA or BODIPYs $\left(r_{1}=18.60 \mathrm{mM}^{-1} \cdot \mathrm{s}^{-1}\right)^{29}$ or a porphyrinic system DPP-ZnP-GdDOTA (see Figure $\mathrm{S} 10 ; \mathrm{r}_{1}=17.9 \mathrm{mM}^{-1} \cdot \mathrm{s}^{-1}$ ) presenting also some aggregation. ${ }^{46}$ Finally, the relaxivity compares well with those of nanosized objects such as MOFs ${ }^{47}$ and micelles ${ }^{48,49}$.

In order to gain more insight into the parameters governing relaxivity, ${ }^{17} \mathrm{O}$ measurements as a function of temperature have been performed. Variable temperature ${ }^{17} \mathrm{O} T_{2}$ measurements give access to the water exchange rate, $k_{\text {ex }}$. The ${ }^{17} O T_{1}$ data are determined by dipole-dipole and quadrupolar relaxation mechanisms and provide information about the rotational correlation time, $\tau_{R}$. The ${ }^{17} O$ chemical shifts give indication of the number of water molecules directly coordinated to $\mathrm{Gd}^{3+}$, $q$. Longitudinal, transverse ${ }^{17} \mathrm{O}$ relaxation rates and chemical shifts were measured as a function of the temperature on aqueous solution of $\mathrm{Gd}_{2}$-Wazaby 6 at $2.39 \mathrm{mM}$, and on a diamagnetic reference $\left(\mathrm{HClO}_{4}, \mathrm{pH} 3.3\right)$ at 9.4 T. Due to the concentration used for the study, ${ }^{17} \mathrm{O}$ chemical shifts and $\mathrm{T}_{1}$ values were too close to those of the reference to yield reliable data. Only ${ }^{17} \mathrm{OT}_{2}$ data were analyzed and are presented in Figure 6. 


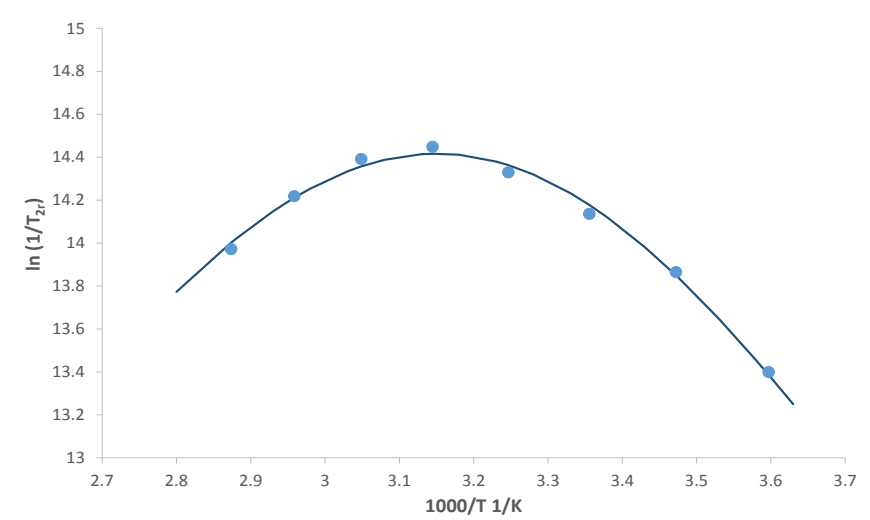

Figure 6: Temperature dependence of the reduced ${ }^{17} \mathrm{O}$ transverse relaxation rates of $\mathrm{Gd}_{2}$-Wazaby6

(2.39 mM) at 9.4 T. The continuous curve represents the best fit to the experimental data points.

The reduced ${ }^{17} \mathrm{O}$ transverse relaxation rates first increase (up to ca. $318 \mathrm{~K}$ ), then decrease with increasing temperature indicating that the complex is in the slow kinetic region at low temperatures and in the fast exchange region at higher temperatures. In the slow kinetic region, $1 / T_{2 r}$ is directly determined by the exchange rate constant $k_{\text {ex }}$, whereas in the fast exchange region, it is determined by the transverse relaxation rate of the coordinated water oxygen, $1 / T_{2 m}$, which is in turn influenced by the water exchange rate, $k_{\mathrm{ex}}$, the longitudinal electronic relaxation rate, $1 / \mathrm{T}_{1 \mathrm{e}}$, and the scalar coupling constant, $\mathrm{A} / \mathrm{h}$.

Due to the different concentrations used for the ${ }^{17} \mathrm{O}$ NMR $\left(4.78 \mathrm{mM}\right.$ of $\left.\mathrm{Gd}^{3+}\right)$ and NMRD samples $\left(1.27 \mathrm{mM}\right.$ of $\left.\mathrm{Gd}^{3+}\right)$ and the aggregation process previously evidenced, the ${ }^{17} \mathrm{O}$ NMR and NMRD data have been analysed separately according to the Solomon-Bloembergen-Morgan theory.

Table 2: Best-fit parameters obtained from the independent fitting of the ${ }^{1} \mathrm{H}$ NMRD profiles (Figure 4) to the SBM theory, including the Lipari-Szabo approach to describe internal flexibility and the transverse ${ }^{17} \mathrm{O} N \mathrm{NR}$ relaxation rates as a function of temperature (Figure 6) at 9.4 T. The number in brackets represents the error on the last digit.

\begin{tabular}{|c|c|c|c|c|}
\hline Parameters & $\begin{array}{c}\text { Gd }_{2^{-}} \\
\text {Wazaby6 }\end{array}$ & {$[\text { DPP-ZnP-GdDOTA }]^{-c}$} & $\begin{array}{c}\text { GdDOTAC }_{12} \\
\text { (micellar form) }^{\mathrm{d}}\end{array}$ & Zn-LZF2Gd ${ }^{e}$ \\
\hline$r_{1}\left(\mathrm{mM}^{-1} \mathrm{~s}^{-1} ; 20 \mathrm{MHz}, 37^{\circ} \mathrm{C}\right)$ & 16.1 & 17.9 & $17.9^{\mathrm{b}}$ & 15.8 \\
\hline$q^{a}$ & 1 & 1 & 1 & 1 \\
\hline$k_{\mathrm{ex}}^{298}\left(10^{6} \mathrm{~s}^{-1}\right)$ & $2.81[ \pm 0.08]$ & 4.1 & 4.9 & 1.6 \\
\hline$\Delta H^{\mp}\left(\mathrm{kJ} \cdot \mathrm{mol}^{-1}\right)$ & $40[ \pm 1]$ & 49.8 & 52 & 40 \\
\hline$\Delta S^{\neq}\left(\mathrm{J} . \mathrm{K}^{-1} \cdot \mathrm{mol}^{-1}\right)$ & $13[ \pm 3]$ & - & +67 & - \\
\hline$E_{l}\left(\mathrm{~kJ} \cdot \mathrm{mol}^{-1}\right)$ & $7[ \pm 4]$ & 40 & 20 & 20 \\
\hline$\tau_{1}^{298}(\mathrm{ps})$ & $111[ \pm 9]$ & 245 & 430 & 145 \\
\hline$E_{\mathrm{g}}\left(\mathrm{kJ} \cdot \mathrm{mol}^{-1}\right)$ & $5 \pm[1.5]$ & 14 & 35 & 13 \\
\hline$\tau_{\mathrm{g}}^{298}(\mathrm{ps})$ & $1840[ \pm 70]$ & 2640 & 1600 & 1160 \\
\hline$S^{2}$ & $0.30[ \pm 0.01]$ & 0.26 & 0.23 & 0.64 \\
\hline
\end{tabular}

${ }^{a}$ fixed in the fit; ${ }^{\mathrm{b}}$ at $60 \mathrm{MHz}$ and $298 \mathrm{~K} ;{ }^{\mathrm{c}}$ From ref ${ }^{46} ;{ }^{\mathrm{d}}$ From ref ${ }^{48}$; ${ }^{\mathrm{e}}$ From ref ${ }^{50}$

In the fitting procedure, the number of water molecules directly coordinated to $\mathrm{Gd}^{3+}$ was fixed to 1 as it is expected for GdDOTAMA complexes. In the fit of the proton relaxivities, the water exchange parameters were fixed to values obtained in the ${ }^{17} \mathrm{O}$ NMR study. The rotational dynamics could be only described by applying the Lipari-Szabo approach. In this approach, that separates local and global motions, characterized by the local and global rotational correlation times, $\tau_{1}^{298}$ and $\tau_{\mathrm{g}}{ }^{298}$, respectively, and a model independent order parameter, $S^{2}$. It reflects the degree of spatial restriction of the local motion with respect to the global motion. Its value ranges from 0 to 1 , with $S^{2}=0$ if the internal motions are isotropic, and $S^{2}=1$ if the internal motions are completely restricted. 
The fitting has been restricted to frequencies above $6 \mathrm{MHz}$ for NMRD data as at low magnetic fields the SBM theory fails in describing electronic parameters and rotational dynamics of slowly rotating species. Details of the analysis and the equations used in the fit are given in the Supporting Information, and the most important parameters obtained are listed in Table 2. A complete list of the parameters fitted for our system is also given Table S1.

For comparison, three other systems have been presented within the table (Table 2): a porphyrinic system with a GdDOTAGA appended (DPP-ZnP-GdDOTA); a GdDOTAC 12 complex in its micellar form, and a 30 aminoacid peptides derived from a $Z n$-finger peptide and bearing a GdDOTAMA complex in a folded form (Zn-bound form; Zn-LZF2Gd). They have been chosen because: (1) they have been all analyzed using the Lipari Szabo approach; (2) the porphyrinic system has a similar molecular weight as our system and shows some small aggregation as well; (3) the GdDOTAC $_{12}$ is in its micellar form which could "mimick" an aggregated state; (4) the peptidic system has a similar coordination sphere for $\mathrm{Gd}^{3+}$ than our system and the relaxivity behavior as a function of temperature is similar (relaxivity limited by rotation rather than water exchange rate).

The fitting of the ${ }^{17} \mathrm{O}$ data yields a value of $k_{\text {ex }}$ of $2.81 .10^{6} \mathrm{~s}^{-1}$, which is lower than that of GdDOTA ( $k_{\text {ex }}$ $=4 \cdot 1 \cdot 10^{6} \mathrm{~s}^{-1}$ ) as expected when a carboxylate function is replaced by an amide function, and in the same range than other GdDOTAMA complexes $\left(c a .2 .10^{6} \mathrm{~s}^{-1}\right) \cdot{ }^{51} \mathrm{It}$ suggests that the aggregation process, which certainly occurs through the BODIPY moiety, does not affect the water exchange rate of the system. The activation entropy calculated is also positive and in the same order of magnitude as that of complexes possessing similar coordination spheres. The water exchange mechanism has not been directly assessed (it is beyond the scope of this study), but the activation entropy calculated gives an indication of a dissociative mechanism as expected for nine-coordinated $\mathrm{Gd}^{3+}$ complexes. In dissociative mechanism, the steric crowding is of primary importance as the determining step is the dissociation of the water molecule. A highly-crowded environment will favour this dissociative activation step. As carboxylate functions are more coordinating than amide function, they imply more constraints explaining why GdDOTA complexes have higher water exchange rates than GdDOTAMA complexes.

The local and global correlation times of the system are within the same range as those of similar systems giving rise to similar relaxivities as shown in Table 2 . The low value of $\mathrm{S}^{2}$, comparable to those of DPP-ZnP-GdDOTA or the micellar system, shows that the motion of the $\mathrm{Gd}^{3+}$-coordinated water proton vector is largely decoupled from the overall motion of the aggregates. This can be explained by the quite flexible chain between the Aza-BODIPY core and the $\mathrm{Gd}^{3+}$ complexes. If we look in more detail, the global correlation time of the system correlates well with that of the micellar system, or that of DPP-ZnP-GdDOTA having a similar molecular weight and presenting some aggregation. It is slightly higher than that of the peptidic system with a higher molecular weight but which is not in an aggregated form. All this confirms that some aggregation occurs for our system. The value of the local correlation time, consistent with that of a small $\mathrm{Gd}^{3+}$ complex, corroborates the flexibility around the $\mathrm{Gd}^{3+}$ complexes and shows that the aggregation certainly occurs through the aza-BODIPY core.

Finally, the temperature dependence of the relaxivity of our system is related to the low water exchange rate of the system together with a slow motion explained by the size of the complex and the presence of some aggregation. The same behavior is not observed with the porphyrinic system due to the difference in the water exchange rates (explained by the different $\mathrm{Gd}^{3+}$ coordination spheres). On the contrary, the peptidic system presents the same (even exacerbated) temperature behavior, which is explained by a more rigid system with slightly lower water exchange rate, despite the same $\mathrm{Gd}^{3+}$ coordination sphere. To sum up, the high relaxivity observed is due to the size of the system and the presence of some small aggregates. 
Phantom images of the system $\mathbf{G d}_{2}$-Wazaby6 have been recorded both in MRI and in fluorescence to highlight its potential.

$\mathrm{T}_{1}$-weighted images were recorded at $7 \mathrm{~T}$, and show a greater contrast compared to the commercial GdDOTA at the same concentration (150 $\mu \mathrm{M})$ (Figure 7).

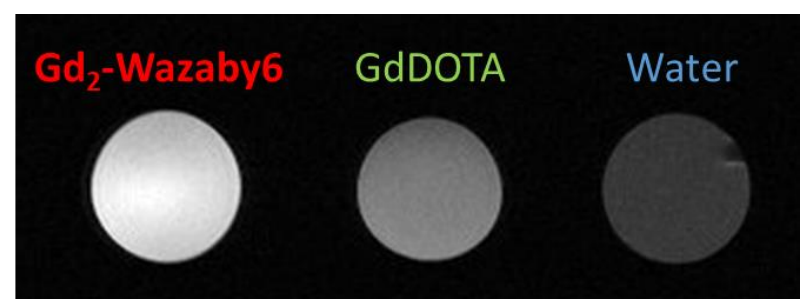

Figure 7: $T_{1}$-weighted MRI phantom images of $\mathrm{Gd}_{2}$-Wazaby6 $(150 \mu \mathrm{M})$, GdDOTA (150 $\left.\mu \mathrm{M}\right)$ and water (reference) at $7 \mathrm{~T}$ using spin-echo sequence with $T E=50 \mathrm{~ms}$ and $T R=500 \mathrm{~ms}$.

Fluorescence images were recorded thanks to an optical imager for in vivo experiments (IVIS Lumina III, Perkin Elmer, Figure 8). Gd $_{2}$-Wazaby6 was imaged at $150 \mu \mathrm{M}$ (the concentration used for MRI imaging), $15 \mu \mathrm{M}$, and $1.5 \mu \mathrm{M}$ in water; Aza-BODIPY 3 was imaged at $150 \mu \mathrm{M}$ as a control. The first observation that can be made is the strong fluorescence of $\mathbf{G d}_{2}$-Wazaby6 with respect to Aza-BODIPY $\mathbf{3}$ ( $\mathbf{3}$ is not fluorescent due to its insolubility in water). Additionally, it is worth noting that $\mathbf{G d}_{2}$-Wazaby $\mathbf{6}$ displays higher fluorescence at $15 \mu \mathrm{M}$ than at $150 \mu \mathrm{M}$, which corroborates the fact that some aggregation phenomenon can occur at high concentration. Moreover, we were pleased to note that a significant signal can be observed up to $850 \mathrm{~nm}$, especially when exciting the molecule at 700 or $720 \mathrm{~nm}$, meaning that $\mathbf{G d}_{2}$-Wazaby6 can be detected wherever we want in biological window.

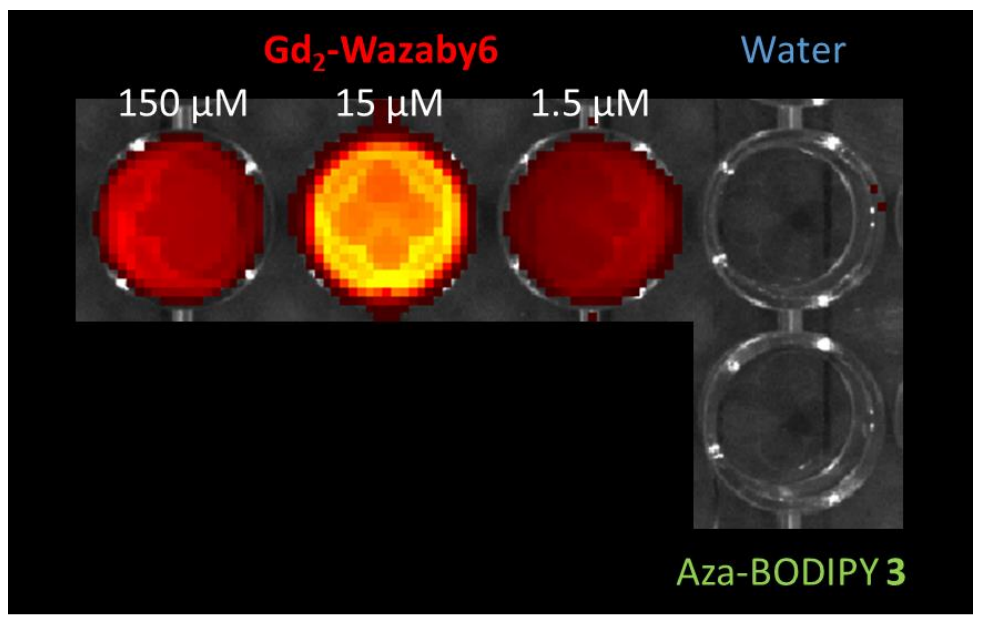

Figure 8: Phantom optical images of of $\mathrm{Gd}_{2}$-Wazaby6 (150 $\mu \mathrm{M}, 15 \mu \mathrm{M}$, and $\left.1.5 \mu \mathrm{M}\right)$, Aza-BODIPY 3 $(150 \mu \mathrm{M})$, and water (reference) excitation at $660 \mathrm{~nm}$ and detection at $710 \mathrm{~nm}$ ).

The relaxivity and the optical emission properties of the system have also been measured in the presence of $0.6 \mathrm{mM}$ of Human Serum Albumin (HSA) (physiological concentration) in order to simulate biological environment. Concerning the relaxivity of $\mathbf{G d}_{2}$-Wazaby6 (at $145 \mu \mathrm{M}$ ), a $14 \%$ increase is observed in water at $\mathrm{pH}=7.3,60 \mathrm{MHz}$, and $25^{\circ} \mathrm{C}$, in the presence of $\mathrm{HSA}$. This increase is explained by the formation of a supramolecular adduct between the probe and HSA as commonly observed for hydrophobic systems. Concerning the optical properties, the emission of Gd2-Wazaby6 (at $2 \mu \mathrm{M}$ ) increased by $230 \%$ in presence of HSA, probably due to the more lipophilic environment. The improved properties of Gd2-Wazaby6 for both MRI and OI in presence of HSA are reassuring regarding its efficiency in real biological media. 
These different preliminary studies are very promising for the future use of our probe for in vivo bimodal imaging.

\section{Conclusion}

For the first time, a monomolecular multimodal imaging probe for MRI and Near-Infrared Fluorescence $\left(\mathbf{G d}_{2}\right.$-Wazaby6) was synthesized thanks to a boron functionalization of an aza-BODIPY. It was fully characterized by usual spectroscopic techniques. The boron functionalization allows good solubility of the system both in water and PBS and enables full photophysical and relaxometric characterizations. Interestingly, the quantum yield of fluorescence of this bimodal system is around $10 \%$ in PBS, which is particularly high for a near infrared fluorophore. Moreover, this probe does not induce the production of singlet oxygen, which could be toxic for biological tissue. The relaxivity observed for $\mathbf{G d}_{2}$-Wazaby6 is high and can be explained both by the size of the system and the presence of some small aggregation process, as also observed in the optical imaging studies. Despite these aggregates the system remains very soluble in water and in biological media, and could be studied without the presence of surfactant as often needed with BODIPY derivatives. Those small aggregates do not imply important fluorescence quenching and enable to achieve high efficiency in MRI (in future work, it will be worth studying whether these small aggregates are still present in vivo). The relaxivity could also be further optimized by rigidifying the system and modifying the coordination sphere of $\mathrm{Gd}^{3+}$, which is the subject of future studies. All together this suggests that these bimodal systems hold great promise as MRI and NIR probes in vitro and in vivo imaging.

\section{Acknowledgments}

The Ministère de l'Enseignement Supérieur et de la Recherche, the Centre National de la Recherche Scientifique (CNRS), the Conseil Régional de Bourgogne (PhD JCE grant \# 2015-9205AAO033S04139 / BG0003203), and the French Research National Agency (ANR) via project JCJC "SPID" ANR-16-CE070020 and project JCJC "WazaBY" ANR-18-CE18-0012 are gratefully acknowledged. This work is part of the projects "Pharmacoimagerie et agents théranostiques" et "Chimie durable, environnement et agroalimentaire" supported by the Université de Bourgogne and the Conseil Régional de Bourgogne through the Plan d'Actions Régional pour l'Innovation (PARI) and the European Union through the PO FEDER-FSE Bourgogne 2014/2020 programs. It was performed within the Dijon's pharmaco-imaging consortium, a regional center of excellence in pharmacoimaging. This work was also supported by a French Government Grant managed by the French National Research Agency (ANR) under the program "Investissements d'Avenir" (reference ANR-10-EQPX-05- 01/IMAPPI Equipex). Chematech ${ }^{\circledR}$ society is warmly thanked for providing DO3A-(tBu$)_{3}-\mathrm{NH}_{2}$ macrocycle. FrenchBIC, GDR AIM, and OncoDesign ${ }^{\circledR}$ are acknowledged for fruitful discussion. LAG acknowledged the French Research National Agency (ANR) for her grant (SADAM ANR-16-CE07-0015-02). Mr Soustelle, and Ms M.-J. Penouilh are gratefully acknowledged for HR-MS, NMR analyses, and Dr Myriam Laly for ionic chromatography.

\section{Supporting Information Available}

This section gathered all experimental details: synthetic procedures, analytical data $\left({ }^{1} \mathrm{H},{ }^{13} \mathrm{C},{ }^{11} \mathrm{~B},{ }^{19} \mathrm{~F}\right.$ NMR, HRMS), ${ }^{1} \mathrm{H}-\mathrm{NMR}$ spectra, analytical and semi-preparative HPLC conditions and chromatograms, ionic chromatography, protocol for determining photophysical properties, absorption, emission and excitation spectra, photophysical experiments with or without HSA and on phantoms, structures of the systems discussed in the article, details concerning relaxometric measurements, temperature dependent ${ }^{17} \mathrm{O}$ measurements, relaxivity measurements in the presence of HAS, phantom MRI images, full parameters used in the fitting procedure of NMRD and ${ }^{17} \mathrm{O}$ data, and equations used for the fits of ${ }^{17} \mathrm{O} N M R$ and ${ }^{1} \mathrm{H}$ NMRD data, report of singlet oxygen formation studies, and details of antiproliferative tests. 
(1) Barabino, G.; Klein, J. P.; Porcheron, J.; Grichine, A.; Coll, J.-L.; Cottier, M. Intraoperative Near-Infrared Fluorescence Imaging Using Indocyanine Green in Colorectal Carcinomatosis Surgery: Proof of Concept. Eur J Surg Oncol 2016, 42 (12), 1931-1937. https://doi.org/10.1016/j.ejso.2016.06.389.

(2) Frangioni, J. V. In Vivo Near-Infrared Fluorescence Imaging. Curr Opin Chem Biol 2003, 7 (5), 626-634.

(3) Kiyose, K.; Aizawa, S.; Sasaki, E.; Kojima, H.; Hanaoka, K.; Terai, T.; Urano, Y.; Nagano, T. Molecular Design Strategies for Near-Infrared Ratiometric Fluorescent Probes Based on the Unique Spectral Properties of Aminocyanines. Chemistry 2009, 15 (36), 9191-9200. https://doi.org/10.1002/chem.200900035.

(4) Yuan, L.; Lin, W.; Zheng, K.; He, L.; Huang, W. Far-Red to near Infrared AnalyteResponsive Fluorescent Probes Based on Organic Fluorophore Platforms for Fluorescence Imaging. Chem Soc Rev 2013, 42 (2), 622-661. https://doi.org/10.1039/c2cs35313j.

(5) Zhu, H.; Fan, J.; Du, J.; Peng, X. Fluorescent Probes for Sensing and Imaging within Specific Cellular Organelles. Acc. Chem. Res. 2016, 49 (10), 2115-2126. https://doi.org/10.1021/acs.accounts.6b00292.

(6) Guo, Z.; Park, S.; Yoon, J.; Shin, I. Recent Progress in the Development of NearInfrared Fluorescent Probes for Bioimaging Applications. Chem. Soc. Rev. 2014, 43 (1), 16-29. https://doi.org/10.1039/C3CS60271K.

(7) Zhao, J.; Chen, J.; Ma, S.; Liu, Q.; Huang, L.; Chen, X.; Lou, K.; Wang, W. Recent Developments in Multimodality Fluorescence Imaging Probes. Acta Pharmaceutica Sinica B 2018, 8 (3), 320-338. https://doi.org/10.1016/j.apsb.2018.03.010.

(8) James, M. L.; Gambhir, S. S. A Molecular Imaging Primer: Modalities, Imaging Agents, and Applications. Physiological Reviews 2012, 92 (2), 897-965. https://doi.org/10.1152/physrev.00049.2010.

(9) Hernot, S.; Manen, L. van; Debie, P.; Mieog, J. S. D.; Vahrmeijer, A. L. Latest Developments in Molecular Tracers for Fluorescence Image-Guided Cancer Surgery. The Lancet Oncology 2019, 20 (7), e354-e367. https://doi.org/10.1016/S14702045(19)30317-1.

(10) Wang, Y.; Song, R.; Feng, H.; Guo, K.; Meng, Q.; Chi, H.; Zhang, R.; Zhang, Z. Visualization of Fluoride Ions In Vivo Using a Gadolinium(III)-Coumarin ComplexBased Fluorescence/MRI Dual-Modal Probe. Sensors 2016, 16 (12), 2165. https://doi.org/10.3390/s16122165.

(11) Wang, Y.; Song, R.; Guo, K.; Meng, Q.; Zhang, R.; Kong, X.; Zhang, Z. A Gadolinium(III) Complex Based Dual-Modal Probe for MRI and Fluorescence Sensing of Fluoride Ions in Aqueous Medium and in Vivo. Dalton Trans. 2016, 45 (44), 1761617623. https://doi.org/10.1039/C6DT02229D.

(12) Harrison, V. S. R.; Carney, C. E.; MacRenaris, K. W.; Waters, E. A.; Meade, T. J. Multimeric Near IR-MR Contrast Agent for Multimodal In Vivo Imaging. J. Am. Chem. Soc. 2015, 137 (28), 9108-9116. https://doi.org/10.1021/jacs.5b04509.

(13) Ceulemans, M.; Nuyts, K.; De Borggraeve, W.; Parac-Vogt, T. Gadolinium(III)-DOTA Complex Functionalized with BODIPY as a Potential Bimodal Contrast Agent for MRI and Optical Imaging. Inorganics 2015, 3 (4), 516-533. https://doi.org/10.3390/inorganics3040516.

(14) Dong, D.; Jing, X.; Zhang, X.; Hu, X.; Wu, Y.; Duan, C. Gadolinium(III)-Fluorescein Complex as a Dual Modal Probe for MRI and Fluorescence Zinc Sensing. Tetrahedron 2012, 68 (1), 306-310. https://doi.org/10.1016/j.tet.2011.10.034. 
(15) Yamane, T.; Hanaoka, K.; Muramatsu, Y.; Tamura, K.; Adachi, Y.; Miyashita, Y.; Hirata, Y.; Nagano, T. Method for Enhancing Cell Penetration of Gd3+-Based MRI Contrast Agents by Conjugation with Hydrophobic Fluorescent Dyes. Bioconjugate Chem. 2011, 22 (11), 2227-2236. https://doi.org/10.1021/bc200127t.

(16) You, Y.; Tomat, E.; Hwang, K.; Atanasijevic, T.; Nam, W.; Jasanoff, A. P.; Lippard, S. J. Manganese Displacement from Zinpyr-1 Allows Zinc Detection by Fluorescence Microscopy and Magnetic Resonance Imaging. Chem. Commun. 2010, 46 (23), 4139 4141. https://doi.org/10.1039/C0CC00179A.

(17) Huynh, A. M.; Müller, A.; Kessler, S. M.; Henrikus, S.; Hoffmann, C.; Kiemer, A. K.; Bücker, A.; Jung, G. Small BODIPY Probes for Combined Dual ${ }^{19} \mathrm{~F}$ MRI and Fluorescence Imaging. ChemMedChem 2016, 11 (14), 1568-1575. https://doi.org/10.1002/cmdc.201600120.

(18) Stasiuk, G. J.; Minuzzi, F.; Sae-Heng, M.; Rivas, C.; Juretschke, H.-P.; Piemonti, L.; Allegrini, P. R.; Laurent, D.; Duckworth, A. R.; Beeby, A.; et al. Dual-Modal Magnetic Resonance/Fluorescent Zinc Probes for Pancreatic $\beta$-Cell Mass Imaging. Chem. Eur. J. 2015, 21 (13), 5023-5033. https://doi.org/10.1002/chem.201406008.

(19) Wang, H.; Mu, X.; Yang, J.; Liang, Y.; Zhang, X.-D.; Ming, D. Brain Imaging with Near-Infrared Fluorophores. Coordination Chemistry Reviews 2019, 380, 550-571. https://doi.org/10.1016/j.ccr.2018.11.003.

(20) Hong, G.; Antaris, A. L.; Dai, H. Near-Infrared Fluorophores for Biomedical Imaging. Nat Biomed Eng 2017, 1 (1), 0010. https://doi.org/10.1038/s41551-016-0010.

(21) Hilderbrand, S. A.; Weissleder, R. Near-Infrared Fluorescence: Application to in Vivo Molecular Imaging. Current Opinion in Chemical Biology 2010, 14 (1), 71-79. https://doi.org/10.1016/j.cbpa.2009.09.029.

(22) Li, J.-B.; Liu, H.-W.; Fu, T.; Wang, R.; Zhang, X.-B.; Tan, W. Recent Progress in Small-Molecule Near-IR Probes for Bioimaging. Trends in Chemistry 2019, 1 (2), 224 234. https://doi.org/10.1016/j.trechm.2019.03.002.

(23) Martinić, I.; Eliseeva, S. V.; Petoud, S. Near-Infrared Emitting Probes for Biological Imaging: Organic Fluorophores, Quantum Dots, Fluorescent Proteins, Lanthanide(III) Complexes and Nanomaterials. Journal of Luminescence 2017, 189, 19-43. https://doi.org/10.1016/j.jlumin.2016.09.058.

(24) Haque, A.; Faizi, Md. S. H.; Rather, J. A.; Khan, M. S. Next Generation NIR Fluorophores for Tumor Imaging and Fluorescence-Guided Surgery: A Review. Bioorganic \& Medicinal Chemistry 2017, 25 (7), 2017-2034. https://doi.org/10.1016/j.bmc.2017.02.061.

(25) Garland, M.; Yim, J. J.; Bogyo, M. A Bright Future for Precision Medicine: Advances in Fluorescent Chemical Probe Design and Their Clinical Application. Cell Chemical Biology 2016, 23 (1), 122-136. https://doi.org/10.1016/j.chembiol.2015.12.003.

(26) Ge, Y.; O'Shea, D. F. Azadipyrromethenes: From Traditional Dye Chemistry to Leading Edge Applications. Chem. Soc. Rev. 2016, 45 (14), 3846-3864. https://doi.org/10.1039/C6CS00200E.

(27) Pliquett, J.; Dubois, A.; Racoeur, C.; Mabrouk, N.; Amor, S.; Lescure, R.; Bettaïeb, A.; Collin, B.; Bernhard, C.; Denat, F.; et al. A Promising Family of Fluorescent WaterSoluble Aza-BODIPY Dyes for in Vivo Molecular Imaging. Bioconjugate Chem. 2019, 30 (4), 1061-1066. https://doi.org/10.1021/acs.bioconjchem.8b00795.

(28) Kim, E.-J.; Bhuniya, S.; Lee, H.; Kim, H. M.; Shin, W. S.; Kim, J. S.; Hong, K. S. In Vivo Tracking of Phagocytic Immune Cells Using a Dual Imaging Probe with Gadolinium-Enhanced MRI and Near-Infrared Fluorescence. ACS Appl. Mater. Interfaces 2016, 8 (16), 10266-10273. https://doi.org/10.1021/acsami.6b03344. 
(29) Fredy, J. W.; Scelle, J.; Guenet, A.; Morel, E.; Adam de Beaumais, S.; Ménand, M.; Marvaud, V.; Bonnet, C. S.; Tóth, E.; Sollogoub, M.; et al. Cyclodextrin Polyrotaxanes as a Highly Modular Platform for the Development of Imaging Agents. Chemistry - A European Journal 2014, 20 (35), 10915-10920. https://doi.org/10.1002/chem.201403635.

(30) Wu, D.; Daly, H. C.; Conroy, E.; Li, B.; Gallagher, W. M.; Cahill, R. A.; O’Shea, D. F. PEGylated BF2-Azadipyrromethene (NIR-AZA) Fluorophores, for Intraoperative Imaging. European Journal of Medicinal Chemistry 2019, 161, 343-353. https://doi.org/10.1016/j.ejmech.2018.10.046.

(31) Yang, Z.; Bai, X.; Ma, S.; Liu, X.; Zhao, S.; Yang, Z. A Benzoxazole Functionalized Fluorescent Probe for Selective $\mathrm{Fe}^{3+}$ Detection and Intracellular Imaging in Living Cells. Anal. Methods 2017, 9 (1), 18-22. https://doi.org/10.1039/C6AY02660E.

(32) Bodio, E.; Denat, F.; Goze, C. BODIPYS and Aza-BODIPYS Derivatives as Promising Fluorophores for in Vivo Molecular Imaging and Theranostic Applications. $J$.

Porphyrins Phthalocyanines 2019, S1088424619501268. https://doi.org/10.1142/S1088424619501268.

(33) Daly, H. C.; Sampedro, G.; Bon, C.; Wu, D.; Ismail, G.; Cahill, R. A.; O’Shea, D. F. BF 2 -Azadipyrromethene NIR-Emissive Fluorophores with Research and Clinical Potential. European Journal of Medicinal Chemistry 2017, 135, 392-400. https://doi.org/10.1016/j.ejmech.2017.04.051.

(34) Grossi, M.; Morgunova, M.; Cheung, S.; Scholz, D.; Conroy, E.; Terrile, M.; Panarella, A.; Simpson, J. C.; Gallagher, W. M.; O’Shea, D. F. Lysosome Triggered Near-Infrared Fluorescence Imaging of Cellular Trafficking Processes in Real Time. Nat Commun 2016, 7 (1), 10855. https://doi.org/10.1038/ncomms10855.

(35) Bellier, Q.; Pégaz, S.; Aronica, C.; Le Guennic, B.; Andraud, C.; Maury, O. NearInfrared Nitrofluorene Substitued Aza-Boron-Dipyrromethenes Dyes. Org. Lett. 2011, 13 (1), 22-25. https://doi.org/10.1021/ol102701v.

(36) Brücher, E.; Tircsó, G.; Baranyai, Z.; Kovács, Z.; Sherry, A. D. Stability and Toxicity of Contrast Agents. In The Chemistry of Contrast Agents in Medical Magnetic Resonance Imaging; John Wiley \& Sons, Ltd, 2013; pp 157-208. https://doi.org/10.1002/9781118503652.ch4.

(37) Wahsner, J.; Gale, E. M.; Rodríguez-Rodríguez, A.; Caravan, P. Chemistry of MRI Contrast Agents: Current Challenges and New Frontiers. Chem. Rev. 2019, 119 (2), 9571057. https://doi.org/10.1021/acs.chemrev.8b00363.

(38) Loudet, A.; Bandichhor, R.; Wu, L.; Burgess, K. Functionalized BF2 Chelated Azadipyrromethene Dyes. Tetrahedron 2008, 64 (17), 3642-3654. https://doi.org/10.1016/j.tet.2008.01.117.

(39) Jing, X.; Yu, F.; Chen, L. Visualization of Nitroxyl (HNO) in Vivo via a LysosomeTargetable near-Infrared Fluorescent Probe. Chem. Commun. 2014, 50 (91), 14253 14256. https://doi.org/10.1039/C4CC07561G.

(40) Bhuniya, S.; Lee, M. H.; Jeon, H. M.; Han, J. H.; Lee, J. H.; Park, N.; Maiti, S.; Kang, C.; Kim, J. S. A Fluorescence off-on Reporter for Real Time Monitoring of Gemcitabine Delivery to the Cancer Cells. Chem. Commun. 2013, 49 (64), 7141-7143. https://doi.org/10.1039/C3CC42653J.

(41) Kamkaew, A.; Burgess, K. Aza-BODIPY Dyes with Enhanced Hydrophilicity. Chem. Commun. 2015, 51 (53), 10664-10667. https://doi.org/10.1039/C5CC03649F.

(42) Daly, H. C.; Sampedro, G.; Bon, C.; Wu, D.; Ismail, G.; Cahill, R. A.; O’Shea, D. F. BF2-Azadipyrromethene NIR-Emissive Fluorophores with Research and Clinical Potential. Eur J Med Chem 2017, 135, 392-400. https://doi.org/10.1016/j.ejmech.2017.04.051. 
(43) Brouwer, A. M. Standards for Photoluminescence Quantum Yield Measurements in Solution (IUPAC Technical Report). Pure and Applied Chemistry 2011, 83 (12), 22132228. https://doi.org/10.1351/PAC-REP-10-09-31.

(44) Karatay, A.; Miser, M. C.; Cui, X.; Küçüköz, B.; Yılmaz, H.; Sevinç, G.; Akhüseyin, E.; Wu, X.; Hayvali, M.; Yaglioglu, H. G.; et al. The Effect of Heavy Atom to Two Photon Absorption Properties and Intersystem Crossing Mechanism in Aza-BoronDipyrromethene Compounds. Dyes and Pigments 2015, 122, 286-294. https://doi.org/10.1016/j.dyepig.2015.07.002.

(45) Singlet Oxygen; 2016; Vol. 1. https://doi.org/10.1039/9781782622208.

(46) Schmitt, J.; Heitz, V.; Sour, A.; Bolze, F.; Kessler, P.; Flamigni, L.; Ventura, B.; Bonnet, C. S.; Tóth, É. A Theranostic Agent Combining a Two-Photon-Absorbing Photosensitizer for Photodynamic Therapy and a Gadolinium(III) Complex for MRI Detection. Chemistry - A European Journal 2016, 22 (8), 2775-2786. https://doi.org/10.1002/chem.201503433.

(47) Carné-Sánchez, A.; Bonnet, C. S.; Imaz, I.; Lorenzo, J.; Tóth, É.; Maspoch, D. Relaxometry Studies of a Highly Stable Nanoscale Metal-Organic Framework Made of $\mathrm{Cu}(\mathrm{II}), \mathrm{Gd}(\mathrm{III})$, and the Macrocyclic DOTP. J. Am. Chem. Soc. 2013, 135 (47), 1771117714. https://doi.org/10.1021/ja4094378.

(48) Nicolle, G. M.; Tóth, É.; Eisenwiener, K.-P.; Mäcke, H. R.; Merbach, A. E. From Monomers to Micelles: Investigation of the Parameters Influencing Proton Relaxivity. $J$ Biol Inorg Chem 2002, 7 (7), 757-769. https://doi.org/10.1007/s00775-002-0353-3.

(49) Martins, A. F.; Morfin, J.-F.; Geraldes, C. F. G. C.; Tóth, É. Gd3+ Complexes Conjugated to Pittsburgh Compound B: Potential MRI Markers of $\beta$-Amyloid Plaques. $J$ Biol Inorg Chem 2014, 19 (2), 281-295. https://doi.org/10.1007/s00775-013-1055-8.

(50) Isaac, M.; Pallier, A.; Szeremeta, F.; Bayle, P.-A.; Barantin, L.; Bonnet, C. S.; Sénèque, O. MRI and Luminescence Detection of $\mathrm{Zn} 2+$ with a Lanthanide Complex-Zinc Finger Peptide Conjugate. Chem. Commun. 2018, 54 (53), 7350-7353. https://doi.org/10.1039/C8CC04366C.

(51) The Chemistry of Contrast Agents in Medical Magnetic Resonance Imaging: Helm/The Chemistry of Contrast Agents in Medical Magnetic Resonance Imaging; Merbach, A., Helm, L., Tóth, É., Eds.; John Wiley \& Sons, Ltd: Chichester, UK, 2013; Vol. Ch.2, p.25. https://doi.org/10.1002/9781118503652.

(52) Raiford, D. S.; Fisk, C. L.; Becker, E. D. Calibration of Methanol and Ethylene Glycol Nuclear Magnetic Resonance Thermometers. Anal. Chem. 1979, 51 (12), 2050-2051. https://doi.org/10.1021/ac50048a040.

(53) Meiboom, S.; Gill, D. Modified Spin- Echo Method for Measuring Nuclear Relaxation Times. Review of Scientific Instruments 1958, 29 (8), 688-691. https://doi.org/10.1063/1.1716296.

(54) Micskei, K.; Helm, L.; Brucher, E.; Merbach, A. E. Oxygen-17 NMR Study of Water Exchange on Gadolinium Polyaminopolyacetates [Gd(DTPA)(H2O)]2- and [Gd(DOTA)(H2O)]- Related to NMR Imaging. Inorg. Chem. 1993, 32 (18), 3844-3850. https://doi.org/10.1021/ic00070a013.

(55) Hugi, A. D.; Helm, L.; Merbach, A. E. Water Exchange on Hexaaquavanadium(III): a Variable-Temperature and Variable-Pressure17O-NMR Study at 1.4 and 4.7 Tesla. Helv. Chim. Acta 1985, 68 (2), 508-521. https://doi.org/10.1002/hlca.19850680224. 


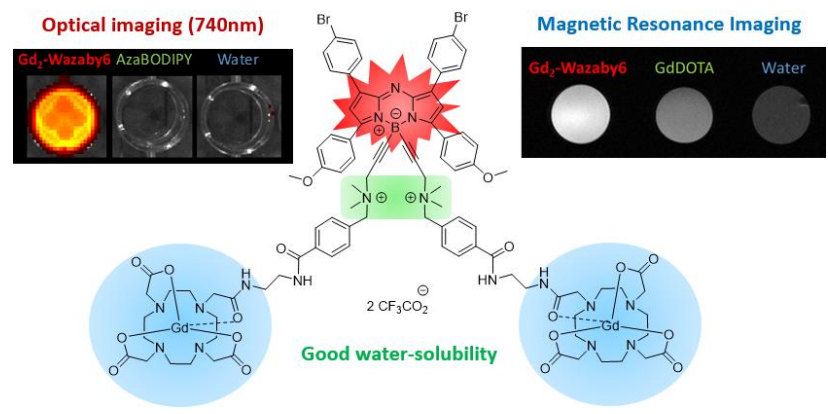

Synthesis and characterization of an original aza-BODIPY system comprising two $\mathrm{Gd}^{3+}$ complexes for magnetic resonance imaging (MRI)/optical imaging (OI) application, by functionalization of the boron center. This is the first aza-BODIPY based bimodal probe displaying a high brightness in near infrared fluorescence $\left(\lambda_{\mathrm{em}}=740 \mathrm{~nm}\right)$, an excellent relaxivity for $\mathrm{MRI}$, and displaying good water solubility. The novel bimodal probe possesses all the requirements for in vivo application. 
